\title{
RIEMANNIAN MANIFOLDS WITH LARGE INVARIANTS
}

\author{
CHRISTOPHER B. CROKE
}

\section{Introduction}

In a previous paper [1] we define some riemannian invariants and discuss their properties. Here we begin the classification of manifolds whose invariants are large. In [1] the invariants were related to the group of isometries of the manifold. In particular, if the group of isometries leaving a point $p \in M$ fixed has large dimension, then the invariants must be large. Hence the results of this paper relate to manifolds with large compact groups acting on them. These manifolds have been studied extensively. See, for example, [3] in the riemannian setting, and [4] in the differentiable setting. The techniques in this paper are geometric in nature, and as such provide completely new proofs as well as generalizations of some of the results in [3] and [4].

This paper follows the notations and conventions of [1]. It also makes extensive use of definitions and results of [1].

In $\$ 1$ we prove some results which will be useful in later sections. This includes classification, up to cohomology ring, of those manifolds such that $\operatorname{dim} N_{S}^{A T C}=1$ for all one-dimensional $S \subset T_{p} M$, and along with $\$ 3$ classification, up to diffeomorphism, of those manifolds such that $\operatorname{dim} N_{S}^{A T C}=2$ for all two-dimensional $S \subset T_{p} M$.

In $\$ 2$ we discuss the case of $A T C_{p}(M) \geqslant \frac{1}{2}(n+3)$. The main purpose here, achieved in Proposition 2.3 and Theorem 2.8, is to find a small dimensional linear subspace $K \subset T_{p} M$ such that all sectional curvatures of sections perpendicular to $K$ are equal.

In $\$ 3$ we classify, up to diffeomorphism, those manifolds $M^{n}$ such that $A T C_{p}(M)=n$ for some $p \in M$. We also study the case $A T C_{p}(M)=n-1$.

In $\$ 4$ we study homogeneous riemannian manifolds such that $A T C_{p}(M) \geqslant \frac{1}{2}(n+3)$. Here we use the subspace $K$, found in $\$ 2$, to define an

Received March 15, 1979. This research was partly supported by the Fannie and John Hertz Foundation. Part of it constitutes part of the author's Ph.D. Dissertaion from the University of Chicago. 
(possibly smaller dimensional) invariant distribution $D$ with nice geometric properties (Theorem 4.1). When $M$ is a normal homogeneous space we show that $D$ is invariant under holonomy so that the universal covering space $\tilde{M}$ splits as a product of a large constant curvature space on another space (Theorem 4.3). We also study, under mild assumptions, what the deck transformations can be (Corollary 4.3). For general homogeneous spaces $M$ such that $A T C_{p}(M) \geqslant \frac{1}{2}(n+3)$ we show that $M$ is the total space of a nice fibration (Proposition 4.7). Finally we return to the case $A T C_{p}(M)=n-1$ in the homogeneous setting (Proposition 4.8).

I would like to thank my advisor, Professor Richard K. Lashof, for his advice and encouragement. I would also like to thank Dr. Allen Back, Professor Melvin Rothenberg, and Dr. Jonathan Sacks for many helpful conversations.

\section{Preliminary results}

In this section we prove some basic results which will be needed in later sections.

1.1. Lemma. For every $S \subset T_{p} M$ we have $d \circ f_{C C}(S)-d(S) \leqslant N-C C_{p}$ (resp. TC, ATC, TG).

Proof. Assume for some $S$ we have $d \circ f_{C C}(S)-d(S)>N-C C_{p}$. Let $S^{\prime}=\left(f_{C C}(S)\right)^{\perp}$ and $\tilde{S}=S \oplus S^{\prime}$. Then we have $d(\tilde{S})=d(S)+d\left(S^{\prime}\right)=$ $d(S)+N-d \circ f_{C C}(S)<d(S)+N-N+C C_{p}-d(S)=C C_{p}$. Now consider $f_{C C}(\tilde{S})$. Since $S \subset \tilde{S}, f_{C C}(S) \subset f_{C C}(\tilde{S})$. Since $\left(f_{C C}(S)\right)^{\perp} \subset \tilde{S},\left(f_{C C}(S)\right)^{\perp}$ $\subset f_{C C}(\tilde{S})$. Thus $f_{C C}(\tilde{S})=T_{p} M$, but $d(\tilde{S})<C C_{p}$, which contradicts the definition of $C C_{p}$. The same proof works for $T C, A T C$, and $T G$.

We next consider the cases where $d \circ f_{C C}(S)=1$ for all one-dimensional $S \subset T_{p} M$, and $d \circ f_{C C}(S)=2$ for all two-dimensional $S \subset T_{p} M$.

1.2. Proposition. Let $M^{n}$ be a complete connected riemannian manifold with $n \geqslant 3$. If there is a $p$ in $M$ such that for all one-dimensional $S \subset T_{p} M$ we have $d \circ f_{C C}(S)=1$ (i.e., $\operatorname{Exp}_{p}(S)=N_{S}^{C C}$ ), then either the tangent cut locus to $p$ is empty (in which case $M$ is diffeomorphic to $\mathbf{R}^{n}$ ) or:

(a) The tangent cut locus to $p$ is a sphere of radius $r$ (for some $r$ ).

(b) The sphere of radius $2 r$ consists entirely of tangent conjugate points of multiplicity $n-1$.

(c) If $V \in T_{p} M$ has $|V|=2 r$, then $\operatorname{Exp}_{p} V=p$.

(d) There are no tangent conjugate points between the sphere of radius $r$ and the sphere of radius $2 r$.

(e) The first tangent conjugate locus is either the sphere of radius $r$ or $2 r$, and all points on this sphere have the same multiplicity. 
(f) If the tangent cut locus is not equal to the first tangent conjugate locus (i.e., the sphere of radius $r$ has not tangent conjugate points), then $M$ is homeomorphic to $\mathbf{R P}^{n}$.

Proof. Since $f_{C C}(S)=S$ for all one-dimensional $S \subset T_{p} M$, we see from Theorem 6.1 of [1] that $N_{S}^{C C}$ is isometric to $N_{S^{\prime}}^{C C}$ for all one-dimensional $S$, $S^{\prime} \subset T_{p} M$. Since $N_{S}^{C C}$ is one-dimensional, it is just the geodesic $\gamma$ with $\gamma^{\prime}(p) \in S$. Thus we see that if some geodesic through $p$ is closed of length $l$, then all geodesics through $p$ are closed of length $l$.

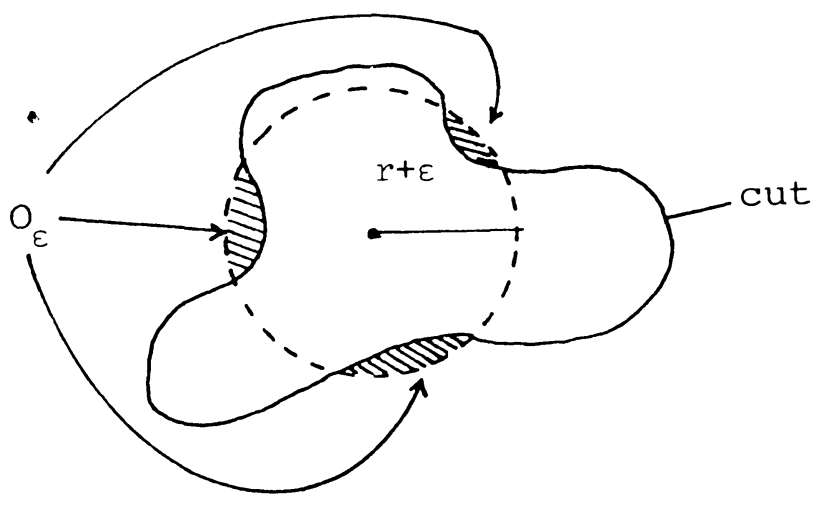

Assume now that the cut locus to $p$ is not empty, and let $r$ be the minimum distance from $p$ to its cut locus. For $\varepsilon>0$ let $0_{\varepsilon}$ be the set $\left\{V \in T_{p} M \mid\|V\|\right.$ $<r+\frac{1}{2} \varepsilon$ and the geodesic $\operatorname{Exp}_{p} t V$ cuts at some $t_{0}<1$ \}. Since $0_{e}$ is an open subset of $T_{p} M$, there is an $X \in 0_{\varepsilon}$ such that $\operatorname{Exp}_{p} X$ does not lie on the cut locus to $p$. (This follows since some $\tilde{X} \in 0_{e}$ is not a critical point of $\operatorname{Exp}_{p}$, and hence there is an open set $U$ with $\tilde{X} \in U \subset 0_{\varepsilon}$ such that $\operatorname{Exp}_{\left.p\right|_{U}}$ is a diffeomorphism onto an open set.) Let $\sigma(t)=\operatorname{Exp}_{p}(t X /\|X\|)$, and let $\tau(t)$ be the unique minimizing geodesic from $p$ to $\operatorname{Exp}_{p} X$. By the definition of $0_{e}, \sigma$ does not minimize so $\sigma \neq \tau$. We also know that the length of $\sigma$ and the length of $\tau$ are less than $r+\frac{1}{2} \varepsilon$. Let $S$ be the one-dimensional subspace spanned by $\sigma^{\prime}(0)$. By assumption $N_{S}^{C C}$ is one-dimensional. Since $N_{S}^{C C}$ is completely convex and $\operatorname{Exp}_{p} X \in N_{S}^{C C}$, we see that $\tau \subset N_{S}^{C C}$. Thus $\sigma$ and $\tau$ fit together to form a closed geodesic $\gamma$ of length $l$. By the definition of $r$ and the above argument we see that $2 r \leqslant l<2 r+\varepsilon$.

Thus we have shown that all geodesics through $p$ are closed of length $l$ where $2 r \leqslant l<2 r+\varepsilon$. But since $\varepsilon$ was arbitrary, $l=2 r$. Now since every geodesic is closed of length $2 r$, they must cut at a length less than or equal to 
$r$. Thus by the definition of $r$ all geodesics cut at a length of $r$. Hence (a) and (c) follow, while (b) follows directly from (c).

Assume there was a tangent conjugate point $V$ such that $r<\|V\|<2 r$. Let $J$ be a Jacobi field along the geodesic $\gamma(t)=\operatorname{Exp}_{p}(t V /\|V\|)$, such that $J(0)=J(\|V\|)=0$. Since $\gamma(2 r)=p$ is a conjugate point of multiplicity $n-1$ and $J(0)=0$ and $\left\langle J(t), \gamma^{\prime}(t)\right\rangle=0$, we have $J(2 r)=0$. Now consider the same Jacobi field along $\tau(t)=\gamma(2 r-t)$. We have $J(0)=0$ and $J(2 r-\|V\|)$ $=0$. So the point $\tau(2 r-\|V\|)$ is conjugate to $p$ along $\tau$. But $2 r-\|V\|<r$ contradicting the definition or $r$. Thus (d) follows.

By (d) we see that any tangent conjugate point on the sphere of radius $r$ is a regular conjugate point. Since the conjugate locus is closed in $T_{p} M$, the intersection with the sphere of radius $r$ is closed. On the other hand the regular conjugate locus consists of a disjoint union of $(n-1)$-dimensional submanifolds (without boundary) each connected component of which consists of conjugate points of the same multiplicity. (For the above results see [6].) Thus (e) follows from (d).

Now assume that no point on the tangent cut locus (the sphere of radius $r$ ) is a conjugate point. Let $r V$ be on the tangent cut locus, and let $q=\operatorname{Exp}_{p} r V$. Since along any minimizing geodesic $p$ is not conjugate to $q$ and since $d(p, q)$ minimizes the distance from $p$ to its cut locus, we see that there are precisely two minimizing geodesics $\gamma, \tau$ from $p$ to $q$ and $\gamma^{\prime}(q)=-\tau^{\prime}(q)$ (see [2, p. 95]). By our previous arguments we see $\gamma(t)=\operatorname{Exp}_{p} t V, \tau(t)=\operatorname{Exp}_{p}-t V$. In particular $r V$ and $-r V$ are the only points $W$ on the cut locus with $\operatorname{Exp}_{p} W=$ $q$. Thus we have shown that $M$ is a disk with its boundary identified through the antipodal map. Thus $M$ is homeomorphic to $\mathbf{R P}^{n}$. The proposition is thus complete.

The following theorem of Warner [7, p. 208] will tell us something about the topology of the manifolds we have just discussed.

1.3. Theorem (Warner). Let $M$ be a compact 1-connected d-dimensional riemannian manifold. If there exists a $p \in M$ for which the first conjugate locus in $T_{p} M$ is a sphere of regular conjugate points of multiplicity $K$, then one of the following holds:

(a) $K=d-1$, and $M$ is homeomorphic to $S^{d}$.

(b) $K=1$ and $d=2 \lambda, \lambda=2,3, \cdots, M$ has the homotopy type of complex projective space.

(c) $K=3$ and $d=4 \lambda, \lambda=2,3, \cdots, M$ has the integral cohomology ring of quaternionic projective space.

(d) $K=7$ and $d=16, M$ has the integral cohomology ring of the projective Cayley Plane.

1.4. Corollary. Let $M^{n}$ be a connected complete riemannian manifold with 
$n \geqslant 3$. If there is a $p \in M$ such that for all one-dimensional $S \subset T_{p} M$ we have $d \circ f_{C C}(S)=1$, then one of the following holds:

(a) $M$ is diffeomorphic to $\mathbf{R}^{n}$.

(b) $M$ is homeomorphic to $\mathbf{R P}^{n}$.

(c) One of the cases of Warner's theorem holds.

Proof. If the tangent cut locus to $p$ is empty, then $M$ is diffeomorphic to $\mathbf{R}^{n}$. If not then it is a sphere of radius $r$, hence $M$ is compact. If no tangent cut point is a conjugate point, then (Prop. 1.2 (f)) $M$ is homeomorphic to $\mathbf{R P}^{n}$. If some tangent cut point is a conjugate point, then (Prop. 1.2(e)) the first conjugate locus is the tangent cut locus and consists of conjugate points of the same multiplicity. Since the tangent cut locus is the first conjugate locus, $M$ is 1-connected. Thus Warner's Theorem gives the result.

Note. If $d \circ f_{A T C}(S)=1$ (resp. TC) for all one-dimensional $S \subset T_{p} M$, then $d \circ f_{C C}(S)=1$ for all one-dimensional $S \subset T_{p} M$. Thus Proposition 1.2, and Corollary 1.4 hold with $C C$ replaced by $A T C$ (resp. TC).

We now consider the case where $d \circ f_{C C}(S)=2$ for all two dimensional $S \subset T_{p} M$.

1.5. Proposition. Let $M^{n}$ be a connected complete riemannian manifold $(n \geqslant 3)$. If there is a $p \in M$ such that all 2-dimensional subspaces $S \subset T_{p} M$ satisfy $d \circ f_{C C}(S)=2$ (resp. $\left.d \circ f_{A T C^{\prime}} d \circ f_{T C}\right)$, then $C C_{p}(M)=n$ (resp. $\left.A T C_{p}, T C_{p}\right)$.

Note. The author does not know whether the result holds for $T G$ or not, but the interesting cases are $A T C$ and $C C$.

Proof. To show $C C_{p}(M)=n$ we need to show that for every linear $S \subset T_{p} M, \operatorname{Exp}_{p} S$ is a topologically closed completely convex almost totally geodesic submanifold of $M$. If $S$ is 1 - or 2-dimensional, this is clear. So assume $\operatorname{dim} S>2$. (Note that we actually need only prove this for $S$ 's such that $\operatorname{dim} S=n-1$, but the proof is the same for all dimensions.) Let $N=\operatorname{Exp}_{p} S$. As in the proof of Proposition 5.2 of [1] it is sufficient to prove that for every $q \in N$ we have:

(1) $\exists S^{q} \subset T_{q} M$ such that $\operatorname{dim} S^{q}=\operatorname{dim} S$ and $\operatorname{Exp}_{q}\left(S^{q}\right) \subset N$,

(2) $\forall q^{\prime} \in N$ if $\gamma$ is a unique minimizing geodesic from $q$ to $q^{\prime}$ such that $q$ is not conjugate to $q^{\prime}$ along $\gamma$, then $\gamma \subset N$.

Let $\tau$ be a geodesic from $p$ to $q$ such that $\tau^{\prime}(0)=V \in S$. We first prove (2). Let $\sigma$ be a geodesic from $p$ to $q^{\prime}$ such that $\sigma^{\prime}(0)=W \in S$. Let $S^{\prime} \subset S$ be the subspace generated by $V$ and $W$. Then $\operatorname{dim} S^{\prime} \leqslant 2$, so $\operatorname{Exp}_{p} S^{\prime}=N_{S^{\prime}}^{C C}$. Since $q$ and $q^{\prime}$ are in $\operatorname{Exp}_{p} S^{\prime}$ and $\operatorname{Exp}_{p} S^{\prime}=N_{S^{\prime}}^{C C}$, we have $\gamma \subset \operatorname{Exp}_{p} S^{\prime} \subset \operatorname{Exp}_{p} S$ $=N$, so (2) holds. To show (1) let $S^{q}$ be the parallel translate of $S$ along $\tau$. Let $Y \in S^{q}$, and let $\tilde{Y}$ be its parallel translate along $\tau$ to $p$. Let $\tilde{S} \subset S$ be the subspace generated by $V$ and $\tilde{Y}$. Then $\operatorname{dim} \tilde{S} \leqslant 2$, so $\operatorname{Exp}_{p} \tilde{S}=N_{\tilde{S}} \mathcal{C}$. In 
particular $q \in N_{\tilde{S}}^{\complement C}$ and $Y \in T_{q} N_{\tilde{S}}^{\complement C}$. Thus $\operatorname{Exp}_{q} Y \in N_{\tilde{S}}^{\complement C}=\operatorname{Exp}_{p} \tilde{S} \subset$ $\operatorname{Exp}_{p} S=N$, and the lemma follows for $C C$. For the convexity conditions $A T C$ and $T C$, the argument used to prove (2) will give the result.

We next consider a result which will serve as an aid in studying the curvature tensor.

1.6. Proposition. Let $S$ be an $r$-dimensional linear subspace of $T_{p} M$ such that for every $(r+1)$-dimensional $S^{\prime}$ with $S \subset S^{\prime}$ we have $d \circ f_{C C}\left(S^{\prime}\right)<$ $d \circ f_{C C}(S)+1$. Let $q \in N_{S}^{C C}$ and $X \in T_{q} N_{S}^{C C}$. Then there exists a number $C_{X}$ such that for every $Y \in\left(T_{q} N_{S}^{C C}\right)^{\perp} \subset T_{q} M$ we have $R(Y, X) X=C_{X} Y$.

Likewise for TG, ATC, and TC.

Proof. Let $\gamma$ be a geodesic from $p$ to $q$ such that $\gamma \subset N_{S}^{C C}$. Let $Y^{\prime} \in T_{p} M$ be the parallel translate of $Y$ along $\gamma$ to $p$. Let $S^{\prime}=S+Y^{\prime}$. Then $S^{\prime}$ has dimension $r+1$. Since $S \subset S^{\prime}$, we have $N_{S}^{C C} \subset N_{S^{\prime}}^{C C}$. By assumption, dimension of $N_{S^{\prime}}^{C C} \leqslant$ dimension of $N_{S}^{C C}+1$. Further since $\gamma \subset N_{S^{\prime}}^{C C}$ and $Y^{\prime} \in$ $T_{p} N_{S^{\prime}}^{C C}$, and $N_{S^{\prime}}^{C C}$ is totally geodesic, we have $Y \in T_{q} N_{S^{\prime}}^{C C}$. Thus $T_{q} N_{S^{\prime}}^{C C}$ is equal to $T_{q} N_{S}^{C C} \oplus Y$, so that $R(Y, X) X \subset T_{q} N_{S^{\prime}}^{C C}$. Further if $Z \in T_{q} N_{S}^{C C}$, we have $\langle R(Y, X) X, Z\rangle=\langle R(Z, X) X, Y\rangle=0$ since $R(Z, X) X \in T_{q} N_{S}^{C C}$ and $Y \in\left(T_{q} N_{S}^{C C}\right)^{\perp}$. Thus $R(Y, X) X=C_{X}^{Y} Y$, which implies that every element of $\left(T_{q} N_{S}^{C C}\right)^{\perp}$ is an eigenvector of $R(-, X) X$, so that they have the same eigenvalue $C_{X}$. Thus $R(Y, X) X=C_{X} Y$, and the proposition follows. The same argument works for TG, ATC, and TC.

\section{Some consequences of $A T C_{p}(M) \geqslant \frac{1}{2}(n+3)$}

In this section we study some of the local and infinitesimal consequences of $A T C_{p}(M) \geqslant \frac{1}{2}(n+3)$. These results will be used extensively in the last two sections. Although all the results in this section are stated in terms of $A T C$, they hold for $T C$ and $C C$. In fact, with the exception of Lemma 2.7 and Corollary 2.9 the results also hold for $T G$.

Definition. Let $M$ be a complete riemannian manifold and $p \in M$, and let $0<\varepsilon$ be some number less than or equal to the distance from $p$ to its cut locus. A linear subspace $Q \subset T_{p} M$ is said to be $\varepsilon$-geodesic if $\operatorname{Exp}_{p}\left(Q \cap B_{\varepsilon}(0)\right)$ is totally geodesic.

2.1. Lemma. If $Q_{j} \rightarrow Q$ where each $Q_{j}$ is an $\varepsilon$-geodesic r-dimensional subspace of $T_{p} M$, then $Q$ is $\varepsilon$-geodesic.

Proof. Since $\varepsilon$ is less than or equal to the distance from $p$ to its cut locus, we know that $\left.\operatorname{Exp}_{p}\right|_{B_{\varepsilon}(0)}$ is a diffeomorphism. Let $q \in N=\operatorname{Exp}_{p}\left(Q \cap B_{e}(0)\right)$ and $X \in T_{q} N$. Let $\tilde{q} \in B_{\varepsilon}(0)$ be the point in $B_{e}(0)$ such that $\operatorname{Exp}_{p}(\tilde{q})=q$. (From now on will be used for this purpose.) Let $\tilde{q}_{j} \rightarrow \tilde{q}$, where $\tilde{q}_{j} \in Q_{j}$, and let $\tilde{X}_{j} \rightarrow \tilde{X}$ where $\tilde{X}_{j} \in T_{\tilde{q}_{j}} Q_{j} \subset T_{\tilde{q}_{j}} T_{p} M$. Let $\gamma_{j}$ be the geodesic determined by 
$X_{j}$, and $\gamma$ the geodesic determined by $X$. Since $X_{j} \rightarrow X, \gamma_{j}(t) \rightarrow \gamma(t)$. For small $t, \gamma_{j}(t) \in \operatorname{Exp}_{p}\left(B_{\varepsilon}(0)\right)$. Thus for small $t, \tilde{\gamma}_{j}(t) \rightarrow \tilde{\gamma}(t)$. Now $\tilde{\gamma}_{j}(t) \in Q_{j}$ so $\tilde{\gamma}(t) \in$ $Q \cap B_{\varepsilon}(0)$. Thus for small $t, \gamma(t) \in N$. Hence $N$ is totally geodesic and the lemma follows.

2.2. Lemma. If $Q$ has the property that for every 2-dimensional linear subspace $T \subset Q$ there is an $\varepsilon$-geodesic $S$ with $T \subset S \subset Q$, then $Q$ is $\varepsilon$-geodesic.

Proof. Let $q \in N=\operatorname{Exp}_{p}\left(Q \cap B_{\varepsilon}(0)\right)$ and let $X \in T_{q} N$. Let $\tilde{q}$ and $\tilde{X}$ be as in the proof of Lemma 2.1. Let $T$ be a 2-dimensional subspace of $T_{p} M$ such that $\tilde{q} \in T$ and $\tilde{X} \in T_{\tilde{q}} T$. By assumption there is an $\varepsilon$-geodesic $S \subset$ $T_{p} M$ with $T \subset S \subset Q$. Since $S$ is $\varepsilon$-geodesic, $N^{S} \equiv \operatorname{Exp}_{p}\left(S \cap B_{e}(0)\right)$ is totally geodesic. Since $S \subset Q, N^{S} \subset N$. Since $\tilde{q} \in T \subset S$ and $\tilde{X} \in T_{\tilde{q}} T \subset$ $T_{\tilde{q}} S$, we have $q \in N^{S}$ and $X \in T_{q} N^{S}$. Since $N^{S}$ is totally geodesic, the geodesic $\gamma$ determined by $X$ lies in $N^{S}$ for small parameter values. Thus for small $t$ we have $\gamma(t) \in N^{S} \subset N$. Therefore $N$ is totally geodesic.

Definition. A linear subspace $S \subset T_{p} M$ is said to be completely $\varepsilon$-geodesic if $S \subset Q$ implies $Q$ is $\varepsilon$-geodesic.

Note. If $S$ is complete $\varepsilon$-geodesic and $S \subset Q$, then $Q$ is completely $\varepsilon$-geodesic.

2.3. Proposition. If $S \subset T_{p} M$ is completely $\varepsilon$-geodesic for some $\varepsilon>0$ then

(i) for every $X \in S$ there is a $C_{X}$ such that $R(Y, X) X=C_{X} Y$ for all $Y \in S^{\perp}$,

(ii) there is a $C$ such that for every $Y_{1}, Y_{2} \in S^{\perp}$, with $\left\langle Y_{i}, Y_{j}\right\rangle=\delta_{i j}$, $R\left(Y_{1}, Y_{2}\right) Y_{2}=C Y_{1}$.

Proof. (i) Since $S+Y$ is $\varepsilon$-geodesic, $R(Y, X) X \in S+Y$. For any $Z \in S$ we have $\langle R(Y, X) X, Z\rangle=\langle R(Z, X) X, Y\rangle=0$ since $R(Z, X) X \in S$ and $Y \in S^{\perp}$. Thus $R(Y, X) X=C_{X}^{Y} Y$. The same argument which we used in Proposition 1.6 now gives (i).

(ii) By the note above $S+Y_{2}$ is completely $\varepsilon$-geodesic. Thus (i) gives $R\left(Y_{1}, Y_{2}\right) Y_{2}=C_{Y_{2}} Y_{1}$. We need only show that $C_{Z_{1}}=C_{Z_{2}}$ for every $Z_{1}, Z_{2} \in$ $S^{\perp}$, with $\left|Z_{1}\right|=\left|Z_{2}\right|=1$. To show this let $s$ be the section determined by $Z_{1}$ and $Z_{2}$ in $T_{p} M$, and let $\bar{Z}_{1}$ be the unit vector in $s$ perpendicular to $Z_{1}$. Then the sectional curvature $K(s)=\left\langle R\left(\bar{Z}_{1}, Z_{1}\right) Z_{1}, \bar{Z}_{1}\right\rangle=\left\langle C_{Z_{1}} \bar{Z}_{1}, \bar{Z}_{1}\right\rangle=C_{Z_{1}}$. Similarly $K(s)=C_{Z_{2}}$, so $C_{Z_{1}}=C_{Z_{2}}$. Thus (ii) follows.

2.4. Proposition. If $S_{1}, S \subset T_{p} M$ are completely $\varepsilon$-geodesic, and $S_{1}+S_{2}$ has codimension at least 2 in $T_{p} M$, then $S_{1} \cap S_{2}$ is completely $\varepsilon$-geodesic.

Proof. By Lemma 2.2 it is sufficient to show that if $Q \subset T_{p} M$ satisfies $S_{1} \cap S_{2} \subset Q$ and $\operatorname{dim} Q \leqslant \operatorname{dim}\left(S_{1} \cap S_{2}\right)+2$, then $Q$ is $\varepsilon$-geodesic. Since $S_{1}+S_{2}$ has codimension $\geqslant 2$, Lemma 2.1 allows us to restrict our attention to those $Q$ 's such that $Q \cap\left(S_{1}+S_{2}\right) \subset S_{1} \cap S_{2}$ (this of course means 
$\left.Q \cap\left(S_{1}+S_{2}\right)=S_{1} \cap S_{2}\right)$. Let $Q$ be as above. Then $Q+S_{1}$ and $Q+S_{2}$ are $\varepsilon$-geodesic. Thus $\left(Q+S_{1}\right) \cap\left(Q+S_{2}\right)$ is $\varepsilon$-geodesic. Since $Q \cap\left(S_{1}+S_{2}\right) \subset$ $S_{1} \cap S_{2}$, we have $\left(Q+S_{1}\right) \cap\left(Q+S_{2}\right)=Q+\left(S_{1} \cap S_{2}\right)=Q$ is $\varepsilon$-geodesic. Thus the proposition follows.

Now consider the function $g_{p}^{A T C}:\{0,1, \cdots, n\} \rightarrow\{0,1, \cdots, n\}$ by $g_{p}^{A T C}(r)=\max \left\{d \circ f_{A T C}(S) \mid S\right.$ is an $r$-dimensional subspace of $\left.T_{p}(M)\right\}$. We can similarly define $g_{p}^{T C}, g_{p}^{C C}$, and $g_{p}^{T G}$.

2.5. Proposition. Assume for some $r \geqslant 1$ that $g_{p}^{A T C}(r+1)=g_{p}^{A T C}(r)+1$, Let $\mathbb{Q}=\left\{S \subset T_{p} M \mid \operatorname{dim} S=r\right.$ and $\left.d \circ f_{A T C}(S)=g_{p}^{A T C}(r)\right\}$. Then if $S \in \mathbb{Q}$, then $f_{A T C}(S)$ is completely $\varepsilon$-geodesic, where $\varepsilon$ is the distance from $p$ to its cut locus.

Proof. Let $S \in \mathbb{Q}$, and let $X_{1}, \cdots, X_{r}$ be a basis for $S$. By Proposition 5.5 of [1] $Q$ is open in $G_{r}(M) \cap \pi^{-1}(p)$. Thus there is an open set $U \subset T_{p} M$ $-\{0\}$ with $X_{1} \in U$ such that if $Y \in U$, then the subspace $S_{Y}=$ $\operatorname{span}\left\{Y, X_{2}, \cdots, X_{r}\right\}$ is in $Q$. Now let $Q$ be a linear subspace of $T_{p} M$ such that $f_{A T C}(S) \subset Q$, and let $U^{\prime}=U \cap Q$.

We claim that $Y \in U^{\prime}$ implies $f_{A T C}\left(S_{Y}\right) \subset Q$. If $Y \in f_{A T C}(S)$, then $S_{Y} \subset$ $f_{A T C}(S)$, so $f_{A T C}\left(S_{Y}\right) \subset f_{A T C}(S) \subset Q$. If $Y \notin f_{A T C}(S)$, then $Y+f_{A T C}(S) \subset$ $f_{A T C}(S+Y)$. For dimension reasons $Y+f_{A T C}(S)=f_{A T C}(S+Y)$. Now $S_{Y}$ $\subset S+Y$, so $f_{A T C}\left(S_{Y}\right) \subset f_{A T C}(S+Y)=Y+f_{A T C}(S) \subset Q$. Thus the claim follows.

Now let $\left.q \in N=\operatorname{Exp}_{p}\left(Q \cap B_{\varepsilon^{\prime}} 0\right)\right)$, and let $\gamma$ be the unique minimizing geodesic from $p$ to $q$. By the definition of $N, \gamma \subset N$. Let $U_{q}^{\prime}$ be the parallel translate of $U^{\prime}$ along $\gamma$.

We claim that $U_{q}^{\prime}$ is an open subset of $T_{q} N$.

For dimension reasons we need only show that $U_{q}^{\prime} \subset T_{q} N$. Let $X=$ $\gamma^{\prime}(0)$ and $Y \in U^{\prime}$. By the same argument used to prove the previous claim, $f_{A T C}\left(S_{Y}+X\right)=f_{A T C}\left(S_{Y}\right)+X$. Thus $f_{A T C}\left(S_{Y}+X\right) \subset Q$. Since $\operatorname{Exp}_{p}\left(f_{A T C}\left(S_{Y}+X\right)\right)=N_{S_{Y}+X}^{A T C}$ is totally geodesic and $X, Y \in f_{A T C}\left(S_{Y}+X\right)$, we see that the parallel translate of $Y$ along $\gamma$ is in $T_{q}\left(\operatorname{Exp}_{p}\left(f_{A T C}\left(S_{Y}+X\right)\right)\right)$ $\subset T_{q} \operatorname{Exp}_{p}(Q)=T_{q} N$. Thus the claim follows.

In the proof of the above claim we showed that if $Y_{q} \in U_{q}^{\prime}$ is the parallel translate of $Y \in U^{\prime}$, then $Y_{q} \in T_{q}\left(N_{S_{Y}+X}^{A T C}\right) \subset T_{q}(N)$. Since $N_{S_{Y}+X}^{A T C}$ is totally geodesic, the geodesic $\gamma_{Y_{q}}$ determined by $Y_{q}$ lies in $N_{S_{Y}+X}^{A T C}$. Thus for small parameter values $\gamma_{Y_{q}}$ lies in $N$. Now if $S^{N}$ is the second fundamental form of $N$, we see that $S^{N}\left(Y_{q}, Y_{q}\right)=0$. Since this is true for all $Y_{q} \in U_{q}^{\prime}$ and $U_{q}^{\prime}$ is open in $T_{q} N$, we see that $S^{N} \equiv 0$. That is, $N$ is totally geodesic. Thus the proposition follows.

2.6. Lemma. If for some $p \in M$ we have $A T C_{p}>\frac{1}{2}(n+3)$, then there is an $r$ satisfying $1 \leqslant r \leqslant n-A T C_{p}+1$ and $g_{p}^{A T C}(r+1)=g_{p}^{A T C}(r)+1 \leqslant n-2$. 
Proof. Assume for all $1 \leqslant r \leqslant n-A T P_{p}+1$ that $g_{p}^{A T C}(r+1)>g_{p}^{A T C}(r)$ +1 . Then for all $r \leqslant n-A T P_{p}+2$ we have

$$
2 r-1 \leqslant g_{p}^{A T C}(r) .
$$

By Lemma 1.1 we have

$$
g_{p}^{A T C}(r)-r \leqslant n-A T P_{p}
$$

Combining (1) and (2) we get

$$
r \leqslant n-A T P_{p}+1 .
$$

But (3) must be true for $r=n-A T P_{p}+2$, a contradiction.

Thus we know that there is an $r$ such that $1 \leqslant r \leqslant n-A T P_{p}+1$ with $g_{p}^{A T C}(r+1)=g_{p}^{A T C}(r)+1$. We need only show that $g_{p}^{A T C}(r)+1 \leqslant n-2$. Let $r$ be the smallest such. Then (1) must hold for $r$, and thus (2) and (3). From (2) and (3) we get

$$
g_{p}^{A T C}(r) \leqslant n-A T P_{p}+r \leqslant 2 n-2 A T P_{p}+1,
$$

from which together with the hypothesis it follows that $g_{p}^{A T C}(r)<2 n-n-3$ $+1=n-2$.

Thus $g_{p}^{A T C}(r)+1 \leqslant n-2$.

2.7. Lemma. If for some $p, A T P_{p}=\frac{1}{2}(n+3)$, then an $r$ exists as in Lemma 7.6 or else

$$
\begin{aligned}
& M \stackrel{\text { diffeo }}{\approx} \mathbf{R}^{n}, \\
& M \stackrel{\text { homeo }}{\approx} S^{n}, \\
& M \stackrel{\text { homeo }}{\approx} \mathbf{R P}^{n} .
\end{aligned}
$$

Proof. We need only consider the proof of Lemma 2.6. Everything follows exactly as before except that in this case we could end up with $g_{p}^{A T C}(r)=n-$ 2. Tracing back the inequalities we see that this can only happen if $g_{p}^{A T C}(s)=$ $2 s-1$ for all $1 \leqslant s \leqslant r$. In particular $g_{p}^{A T C}(1)=1$. Further since $A T P_{p}=$ $\frac{1}{2}(n+3)$, we know that $n$ is odd. Hence Corollary 1.4 gives the result.

2.8. Theorem. Let $M$ be a complete connected $n$-dimensional riemannian manifold. If for some $p \in M, A T P_{p}(M)>\frac{1}{2}(n+3)$, then there is a subspace $K \subset T_{p} M$ such that

(i) $K$ is uniquely determined by the metric,

(ii) $\operatorname{Exp}_{p} K=N_{K}^{A T C}$, 
(iii) $K$ is completely $\varepsilon$-geodesic where $\varepsilon$ is the distance from $p$ to its cut locus,

(iv) $\operatorname{dim} K \leqslant n-A T P_{p}$.

Proof. By Lemma 2.6 there is an $r, 1 \leqslant r \leqslant A T P_{p}+1$, such that $g_{p}^{A T C}(r+1)=g_{p}^{A T C}(r)+1 \leqslant n-2$. Let $r_{0}$ be the smallest such $r$. Let $\mathbb{Q}=$ $\left\{S \subset T_{p} M \mid \operatorname{dim} S=r_{0}\right.$ and $\left.d \circ f_{A T C}(S)=g_{p}^{A T C}\left(r_{0}\right)\right\}$, and $K=\cap_{S \in \mathbb{Q}} f_{A T C}(S)$. Properties (i) and (ii) are clear from the definition.

We claim that for every $S \in \mathbb{Q}$ there is a $Q^{S} \subset T_{p} M$ such that $K \subset Q^{S} \subset$ $f_{A T C}(S), S \cap Q^{S}=\{0\}$, and $Q^{S}$ is completely $\varepsilon$-geodesic.

By Proposition $2.5, f_{A T C}(S)$ is completely $\varepsilon$-geodesic. Let $X \in S$, and choose $X_{2}, X_{3}, \cdots, X_{r_{0}} \in S$ such that $X, X_{2}, \cdots, X_{r_{0}}$ form a basis of $S$. Choose $Y$ near $X$ such that $Y \notin f_{A T C}(S)$, and $S^{X}=\operatorname{span}\left\{Y, X_{2}, \cdots, X_{r_{0}}\right\}$ is in $Q$. This can be done since $\mathbb{Q}$ is open by Proposition 5.5 of [1]. Since $g_{p}^{A T C}\left(r_{0}+1\right)=g_{p}^{A T C}\left(r_{0}\right)+1, f_{A T C}(S)+f_{A T C}\left(S^{X}\right) \subset f_{A T C}\left(S+S^{X}\right)=$ $f_{A T C}(S)+Y$, which has dimension $g_{p}^{A T C}(r)+1 \leqslant n-2$, by Lemma 7.8. Now by Proposition 2.4, $Q_{1}^{S} \equiv f_{A T C}(S) \cap f_{A T C}\left(S^{Y}\right)$ is completely $\varepsilon$-geodesic. Further, $K \subset Q_{1}^{S}$ by the definition of $K$. Now $X \notin Q_{1}^{S}$ because $X \notin$ $f_{A T C}\left(S^{Y}\right) . X \notin f_{A T C}\left(S^{Y}\right)$, for if it were $S \subset f_{A T C}\left(S^{Y}\right)$ and thus for dimension reasons $f_{A T C}(S)=f_{A T C}\left(S^{Y}\right)$, but $Y \in f_{A T C}\left(S^{Y}\right)$ and $Y \notin f_{A T C}(S)$.

Now let $X^{\prime} \in S \cap Q_{1}^{S}$, and $Q_{2}^{S}=Q_{1}^{S} \cap f_{A T C}\left(S^{X^{\prime}}\right)$ where $S^{X^{\prime}}$ is defined similarly to $S^{X}$. For dimension reasons after a finite number of steps $Q_{i}^{S} \cap S=\{0\}$. Let $Q^{S}=Q_{i}^{S}$. Then $Q^{S} \cap S=\{0\}, K \subset Q^{S}$, and $Q^{S}$ is completely $\varepsilon$-geodesic. Thus the claim follows.

Property (iv) follows as $\operatorname{dim} K \leqslant \operatorname{dim} Q^{S} \leqslant \operatorname{dim} f_{A T C}(S)-\operatorname{dim} S \leqslant n-$ $A T P_{p}$, by Lemma 1.1 .

For property (iii) one need only note that for dimension reasons $K$ can be achieved as a finite intersection $Q^{S_{1}} \cap Q^{S_{2}} \cap \cdots \cap Q^{S_{m}}$. Since $\operatorname{dim}\left(Q^{S_{i}}+\right.$ $\left.Q^{S_{j}}\right) \leqslant \operatorname{dim} Q^{S_{i}}+\operatorname{dim} Q^{S_{j}} \leqslant 2 n-2 A T P_{p} \leqslant n-3$, we see by Lemma 2.4 that $K$ is completely $\varepsilon$-geodesic.

2.9. Corollary. If $\operatorname{ATP}_{p}(M)=\frac{1}{2}(n+3)$, then a subspace $K$ as in Theorem 2.8 exists or

$$
\begin{aligned}
& M \stackrel{\text { diffeo }}{\approx} \mathbf{R}^{n}, \\
& M \stackrel{\text { homeo }}{\approx} S^{n}, \\
& M \stackrel{\text { homeo }}{\approx} \mathbf{R P}^{n} .
\end{aligned}
$$

Proof. This follows from Lemma 2.7.

Remark. This Theorem is most powerful when combined with Proposition 2.3. We see that all sections perpendicular to $K$ have the same sectional curvature. 


\section{Manifolds with largest invariants}

In this section we study manifolds whose invariants are maximal or one less than maximal (i.e., $T G_{p}(M)=n$ or $n-1$ resp. $T C, A T C, C C$ ).

From $\S 1$ of [1] we have $T C_{p} \leqslant A T P_{p} \leqslant C C_{p} \leqslant T G_{p} \leqslant n$. Thus we have $T C_{p}=n \Rightarrow A T C_{p}=n \Rightarrow C C_{p}=N \Rightarrow T G_{n}=n$. Therefore we first study the case where $T G_{p}=n$.

3.1. Theorem. If $M^{n}(n \geqslant 3)$ is a connected complete riemannian manifold such that at every point $p \in M$ we have $T G_{p}(M)=n$, then $M$ is a space of constant curvature.

Proof. First fix $p \in M$. By Lemma 1.1, $\operatorname{Exp}_{p} S=N_{S}^{T G}$ for every linear subspace $S \subset T_{p} M$. In particular every $S \subset T_{p} M$ is $\varepsilon$-geodesic where $\varepsilon$ is the distance from $p$ to its cut locus. Thus the subspace $\{0\} \subset T_{p} M$ is completely $\varepsilon$-geodesic. Now Proposition 2.3 tells us that all sections at $p$ have the same sectional curvature. Since $n \geqslant 3$, Shur's theorem gives the result.

3.2. Theorem. If $M$ is a connected simply connected complete riemannian manifold such that $T G_{p}(M)=n$ for some point $p \in M$, then $M$ is diffeomorphic to $\mathbf{R}^{n}$ or $S^{n}$.

Proof. For $n=2$ the result is obvious, so we will assume $n \geqslant 3$. If the first conjugate locus to $p$ is empty, then $\operatorname{Exp}_{p}: T_{p} M \rightarrow M$ is a covering. Since $M$ is simply connected, $M$ is diffeomorphic to $\mathbf{R}^{n}$. Therefore we assume that the first conjugate locus is not empty.

We claim that the conjugate locus consists entirely of conjugate points of multiplicity $n-1$.

To show this let $\gamma$ be a geodesic emanating from $p$. Consider the Jacobi equation for Jacobi fields perpendicular to $\gamma$, i.e.,

$$
\nabla_{T}^{2} J+R(J, T) T=0, \quad T=\gamma^{\prime}(t) .
$$

By Lemma 1.1 we see that $d \circ f_{T G}(S)=1$ for all 1-dimensional $S \subset T_{p} M$ while $d \circ f_{C C}(S)=2$ for all 2-dimensional $S \subset T_{p} M$. Thus by Proposition 1.6 we see $R(J, T) T=c(t) J$, where $c$ is a function defined along $\gamma$, which reduces (*) to $\nabla_{T}^{2} J+c(t) J=0$. Therefore there is a function $f(t)$ with $f(0)=0$ such that all Jacobi fields $J$ along $\gamma$ such that $J(0)=0$ and $\left\langle J(t), \gamma^{\prime}(t)\right\rangle=0$ have the form $f(t) X$, where $X$ is a parallel field along $\gamma$. Thus if one such Jacobi field vanishes at $t_{0}$, then $f\left(t_{0}\right)=0$ so all such Jacobi fields vanish at $t_{0}$. Hence the claim.

We also claim that the conjugate locus in $T_{p} M$ consists of concentric spheres of constant radius.

Since all conjugate points have the same multiplicity $n-1$, the conjugate locus in $T_{p} M \cdot$ consists entirely of regular conjugate points. Warner [6] shows that the regular conjugate locus consists of disjoint $(n-1)$-dimensional 
submanifolds of $T_{p} M$. Since the conjugate locus is closed, each connected component is closed. Warner further shows [6] that if $V$ is a regular conjugate point of multiplicity $\geqslant 2$, then the nullity of the exponential map at $V$ is contained in the tangent space to the submanifold of conjugate points passing through $V$. In our case all conjugate points have multiplicity $n-1 \geqslant 2$, and the nullity of the exponential map is precisely the space perpendicular to the ray from the origin of $T_{p} M$.

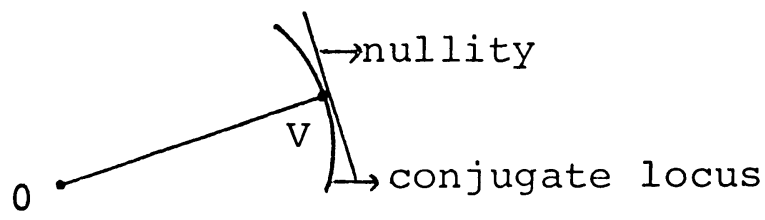

This forces the conjugate locus to consist of concentric spheres of constant radius.

Since $M$ is simply connected and the first conjugate locus consists entirely of conjugate points of order $n-1 \geqslant 2$, Lemma 2.9 of [1] tells us that the first conjugate locus is equal to the cut locus. Since the cut locus is a sphere of conjugate points of multiplicity $n-1$, the image under $\operatorname{Exp}_{p}$ will be a single point $q \in M$. Since $M$ is a disk with its boundary identified to a point, $M$ is homeomorphic to $S^{n}$.

We now show that $M$ is diffeomorphic to $S^{n}$. We have shown above that there is a number $l$ such that every geodesic from $p$ hits $q$ at a distance of precisely $l$. We will explicitly demonstrate a diffeomorphism from the sphere $S^{n}$ of constant curvature with diameter $l$ to $M$.

Consider the function $\varphi: T_{p} M \rightarrow T_{q} M$ defined as follows: for $V \in T_{p} M$ let $\gamma_{V}$ be the geodesic with tangent vector $V$, and define $\varphi(V)$ to be $-\gamma_{V}^{\prime}(q)$. Let $\varphi(0)=0$. It is clear that $\varphi$ is a norm-preserving continuous function. We will show that $\varphi$ is orthogonal. Since $\varphi$ is norm-preserving, it is sufficient to show $\varphi$ is linear.

Step 1. If $\langle X, Y\rangle=0$ for $X, Y \in T_{p} M$, then $\langle\varphi(X), \varphi(Y)\rangle=0$.

Since $\operatorname{dim} M \geqslant 3$, we can choose $Z \in T_{p} M$ such that $\langle Z, X\rangle=\langle Z, Y\rangle$ $=0$. Since $T G_{p}(M)=n$, Lemma 1.1 tells us that for every linear subspace $S \subset T_{p} M, \operatorname{Exp}_{p} S$ is a topologically closed totally geodesic submanifold. Let $N_{X Y}, N_{X Z}, N_{Y Z}$, and $N$ be the submanifolds corresponding to the spans of $X$ and $Y, X$ and $Z, Y$ and $Z$, and $X, Y$, and $Z$ respectively. We have $\operatorname{dim} N_{X Y}$ $=\operatorname{dim} N_{X Z}=\operatorname{dim} N_{Y Z}=2$ and $\operatorname{dim} N=3$. Further $N_{X Y}, N_{X Z}, N_{Y Z} \subset N$. Let $\gamma_{X}, \gamma_{Y}$, and $\gamma_{Z}$ be the geodesics corresponding to $X, Y$, and $Z$ respectively. 
Let $\tilde{X}, \tilde{Y}$, and $\tilde{Z} \in T_{q} N \subset T_{q} M$ be the parallel translates of $X, Y$, and $Z$ along $\gamma_{y}$ to $q$ respectively. Since parallel translation preserves the metric,

$$
\langle\tilde{X}, \tilde{Y}\rangle=\langle\tilde{X}, \tilde{Z}\rangle=\langle\tilde{Y}, \tilde{Z}\rangle=0 \text {. }
$$

By the definition of $\varphi, \tilde{Y}=-\varphi(Y)$ so

$$
\langle\tilde{X}, \varphi(Y)\rangle=0 \text {. }
$$

Since $N_{X Y}$ and $N_{Y Z}$ are totally geodesic and $\gamma_{y} \subset N_{X Y} \cap N_{Y Z}$, we have $\tilde{X} \in T_{q} N_{X Y}, \tilde{Z} \in T_{q} N_{Y Z}, \tilde{Y} \in T_{q} N_{X Y} \cap T_{q} N_{Y Z}$, in particular,

$$
\tilde{X} \in T_{q} N_{X Y} \text { and } \tilde{X} \in\left(T_{q} N_{Y Z}\right)^{\perp} \text {. }
$$

Now let $\tilde{\tilde{X}}$, $\tilde{\tilde{Y}}$, and $\tilde{\tilde{Z}}$ be the corresponding parallel translates along $\gamma_{Z}$ to $q$. Just as above we get

$$
\tilde{\tilde{X}} \in T_{q} N_{X Z} \text { and } \tilde{\tilde{X}} \in\left(T_{q} N_{Y Z}\right)^{\perp} .
$$

Since $\tilde{X}, \tilde{\tilde{X}} \in T_{q} N$ of dimension 3 and both are perpendicular to $T_{q} N_{Y Z}$ of dimension 2, we have $\tilde{X}= \pm \tilde{X}$. In particular, (iii') tells us that $\tilde{X} \in T_{q} N_{X Z}$, while (iii) gives $\tilde{X} \in T_{q} N_{X Y}$. Therefore $\tilde{X} \in T_{q} N_{X Z} \cap T_{q} N_{X Y}=T_{q}\left(\gamma_{X}\right)$, which implies that $\tilde{X}= \pm \varphi(X)$. Hence (ii) tells us that $\langle\varphi(X), \varphi(Y)\rangle=0$, and Step 1 is complete.

Step 2. For every $X, Y \in T_{p} M,\langle X, Y\rangle= \pm\langle\varphi(X), \varphi(Y)\rangle$.

As before choose $Z \in T_{p} M$ such that $\langle Z, X\rangle=\langle Z, Y\rangle=0$. Let $\tilde{X}, \tilde{Y}, \tilde{Z}$ be the corresponding parallel translates of $X, Y, Z$ along $\gamma_{Z}$. Now $\langle\tilde{X}, \tilde{Y}\rangle=$ $\langle X, Y\rangle$ and $\langle\tilde{Z}, \tilde{X}\rangle=\langle\tilde{Z}, \tilde{Y}\rangle=0$. Further as before we have $\tilde{Z}=-\varphi(Z)$, $\tilde{X} \in T_{q} N_{X Z}$, and $\tilde{Y} \in T_{q} N_{Y Z}$. By Step $1,\langle\varphi(Z), \varphi(Y)\rangle=\langle\varphi(Z), \varphi(X)\rangle=$ 0. Since $\varphi(X), \varphi(Y) \in T_{q} N_{X Y}$ we have $T_{q} N_{X Y}=\varphi(Z)^{\perp} \cap T_{q} N$. Since $\tilde{Z}=-$ $\varphi(Z)$, we have $\tilde{X} \in T_{q} N_{X Y}$ and $\tilde{Y} \in T_{q} N_{X Y}$. Thus $\tilde{X} \in T_{q} N_{X Y} \cap T_{q} N_{X Z}=$ $T_{q}\left(\gamma_{X}\right)$ and $\tilde{Y} \in T_{q} N_{Y Z} \cap T_{q} N_{X Y}=T_{q}\left(\gamma_{Y}\right)$, which imply that $\tilde{X}= \pm \varphi(X)$ and $\tilde{Y}= \pm \varphi(Y)$. So $\pm\langle\varphi(X), \varphi(Y)\rangle=\langle\tilde{X}, \tilde{Y}\rangle=\langle X, Y\rangle$, and Step 2 follows.

Now we complete the proof that $\varphi$ is linear. By the definition of $\varphi$ we have $\varphi(a X)=a \varphi(X)$. Consider $\varphi(X+Y)$. Since $X+Y$ is contained in the plane spanned by $X$ and $Y, \varphi(X+Y) \in T_{q} N_{X Y} . T_{q} N_{X Y}$ is also the plane spanned by $\varphi(X)$ and $\varphi(Y)$. Now since $\varphi$ preserves norms and is continuous, Step 2 tells us that $\varphi(X+Y)=\varphi(X)+\varphi(Y)$.

Now let $S^{n}$ be the constant curvature sphere of diameter $l$. Choose antipodal points $\tilde{p}$ and $\tilde{q}$ in $S^{n}$, and let $\tilde{\varphi}: T_{\tilde{p}} S^{n} \rightarrow T_{\tilde{q}} S^{n}$ be the corresponding function. Let $\psi$ be an isometry from $T_{\tilde{p}} S^{n}$ to $T_{p} M$. Define an isometry $\psi^{\prime}: T_{\tilde{q}} S^{n} \rightarrow T_{q} M$ by $\psi^{\prime}=\varphi \circ \psi \circ \tilde{\varphi}^{-1}$. We have the following commutative diagram of diffeomorphism: 


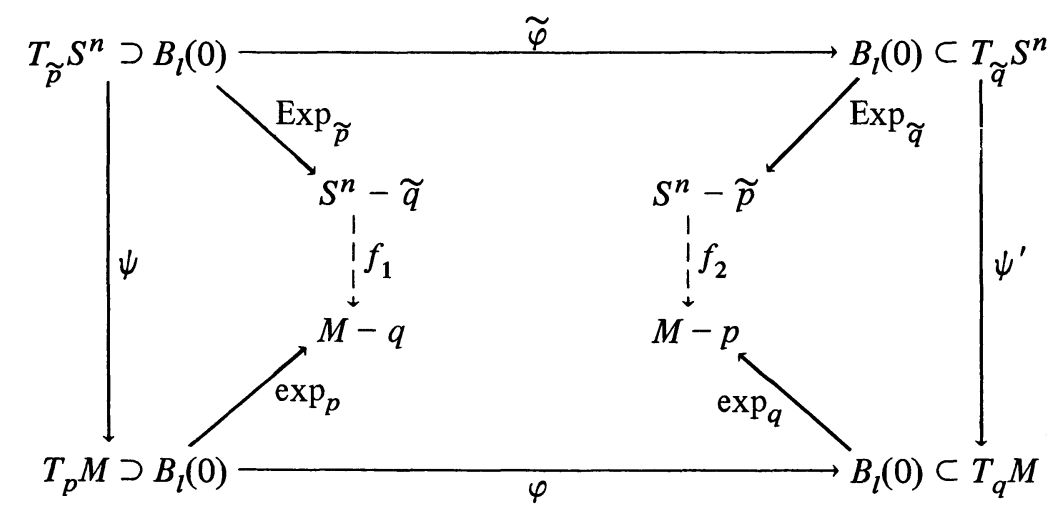

where

$$
f_{1}=\operatorname{Exp}_{p} \circ \psi \circ\left(\operatorname{Exp}_{\tilde{p}}\right)^{-1}, f_{2}=\operatorname{Exp}_{q} \circ \psi^{\prime} \circ\left(\operatorname{Exp}_{\tilde{q}}\right)^{-1}
$$

The proof will be complete when we show that $f_{1}=f_{2}$ on $S^{n}-\{\tilde{p}, \tilde{q}\}$. Let $x \in S^{n}-\{\tilde{p}, \tilde{q}\}$. There is a unique geodesic $\gamma$ from $\tilde{p}$ to $\tilde{q}$ such that $x \in \gamma$. Let $V$ be the unit tangent vector to $\gamma$ at $p$. Then $\left(\operatorname{Exp}_{\tilde{p}}\right)^{-1}(x)=a V$ for some constant $a$, while $\left(\operatorname{Exp}_{\tilde{q}}\right)^{-1}(x)=(l-a) \tilde{\varphi}(V)$. Now $f_{2}(x)=\operatorname{Exp}_{q}((l-$ $\left.a) \psi^{\prime} \tilde{\varphi}(V)\right)=\operatorname{Exp}_{q}((l-a) \varphi \psi(V))=\operatorname{Exp}_{p} a \psi(V)=\operatorname{Exp}_{p}(\psi(a V))=f_{1}(x)$. Thus the theorem follows. q.e.d.

If we make the stronger assumption that $C C_{p}(M)=n$ for some $p \in M$, then we can drop the simply-connected assumption and get

3.3. Theorem. Let $M^{n}$ be a connected complete riemannian manifold such that $C_{p}(M)=n$ for some $p \in M$. Then $M$ is diffeomorphic to $\mathbf{R}^{n}, S^{n}$ or $\mathbf{R P}^{n}$.

Proof. Since $C C_{p}(M)=n$ implies $T G_{p}(M)=n$, Theorem 3.2 gives the result in the case that $M$ is simply connected. Thus we assume that $M$ is not simply connected. Let $\tilde{M} \stackrel{\pi}{\rightarrow} M$ be the universal covering space. Now by Proposition 3.6 of [1], $T G_{p}(\tilde{M}) \geqslant T G_{\pi}(\tilde{M})(M)=n$. So $\tilde{M}$ is diffeomorphic to $S^{n}$ or $\mathbf{R}^{n}$. Now since $C C_{p}(M)=n$, Lemma 1.1 tells us that for all 1-dimensional $S \subset T_{p} M, d \circ f_{C C}(S)=1$. Since $M$ is not simply connected, the cut locus to $p$ in $T_{p} M$ is not empty and is not equal to the first conjugate locus. Thus Proposition 1.2 gives that $M$ is homeomorphic to $\mathbf{R P}^{n}$. The proof to Proposition 1.2 also tells us what the map $\pi: \tilde{M} \rightarrow M$ is. Studying this one sees that there is a function $f$ such that the diagram

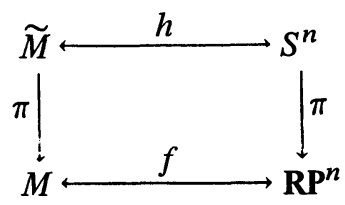


commutes, where $h$ is the diffeomorphism constructed in Theorem 3.2. thus $f$ is a diffeomorhism and the result follows. q.e.d.

Now since $A T C_{p}(M)=n$ implies $C C_{p}(M)=n$ and since $T C_{p}(M)=n$ implies $A T C_{p}(M)=n$, we are able to complete the case where the invariants are $n$.

Let $M^{n}$ be a complete connected riemannian manifold. Then the following hold.

(a) If $T G_{p}(M)=n$ for all $p \in M$, then $M$ has constant curvature.

(b) If $T G_{p}(M)=n$ for some $p \in M$ and $M$ is 1 -connected, then $M \stackrel{\text { diffeo }}{\approx} S^{n}$, or $\mathbf{R}^{n}$.

(c) If $C C_{p}(M)=n$ for some $p \in M$, then $M \stackrel{\text { diffeo }}{\approx} \mathbf{R}^{n}, S^{n}$, or $\mathbf{R} \mathbf{P}^{n}$.

(d) If $A T C_{p}(M)=n$ for some $p \in M$, then $M \stackrel{\text { diffeo }}{\approx} \mathbf{R}^{n}, S^{n}$, or $\mathbf{R P}^{n}$.

(e) If $T C_{p}(M)=n$ for some $p \in M$, then $M \stackrel{\text { diffeo }}{\simeq} \mathbf{R}^{n}$.

In terms of the differential invariants defined in [1] we have; for a smooth connected manifold $M$,

(b) $T G(M)=n$ and $M$ 1-connected $\Rightarrow M \stackrel{\text { diffeo }}{\simeq} \mathbf{R}^{n}$ or $S^{n}$;

(c) $C C(M)=n \Rightarrow M \stackrel{\text { diffeo }}{\simeq} \mathbf{R}^{n}, S^{n}$ or $\mathbf{R P}^{n}$;

(d) $A T C(M)=n \Rightarrow M \stackrel{\text { diffeo }}{\simeq} \mathbf{R}^{n}, S^{n}$ or $\mathbf{R} \mathbf{P}^{n}$;

(e') $T C(M)=N \Rightarrow M \stackrel{\text { diffeo }}{\simeq} \mathbf{R}^{n}$.

In order to see how this relates to isometry groups, we first note that if $n \geqslant 4$ and $S O(n)$ acts as isometries leaving $p \in M^{n}$ fixed, then $A T C_{p}(M)=$ $n$. From the inequality of $[1, \S 1]$ it follows that $A T C_{p}(M)=n$ or $n-1$. However, since every 2-dimensional subspace $S \subset T_{p} M$ is left fixed by some $S O(n-2) \subset S O(n)$, we see that $d \circ f_{C C}(S)=2$. Thus Proposition 1.5 gives $A T C_{p}(M)=n$.

Combining the above results we obtain the classical result:

3.4. Corollary. If the isometry group of a connected complete riemannian manifold $M^{n}$ has dimension $\frac{1}{2} n(n+1)$, then $M$ is a space of constant curvature diffeomorphic to $\mathbf{R}^{n}, S^{n}$ or $\mathbf{R P}^{n}$.

We also get

3.5. Corollary. If $S O(n)$ acts on a smooth connected manifold $M^{n}$ as a group of diffeomorphisms leaving a point $p$ fixed, then $M$ is diffeomorphic to $\mathbf{R}^{n}, S^{n}$ or $\mathbf{R P}^{n}$.

We now consider the $(n-1)$-case.

3.6. Theorem. Let $M^{n}$ be a connected complete riemannian manifold ( $n \geqslant$ 4). If $A T C_{p}(M)=n-1$ for some $p \in M$, then one of the following holds:

(1) For every 1-dimensional $S \subset T_{p} M$ we have $d \circ f_{A T C}(S)=1$, in which case:

(a) $M$ is diffeomorphic to $\mathbf{R}^{n}$, 
(b) $M$ is homeomorphic to $S^{n}$,or

(c) $M$ is homeomorphic to $\mathbf{R P}^{n}$.

(2) For some 1-dimensional $S \subset T_{p} M, d \circ f_{A T C}(S)=2$, in which case:

(a) the universal covering space is $n-1$ connected, or

(b) there is a 1-dimensional almost totally convex topologically closed totally geodesic submanifold $N$ through $p$ such that if $q, q^{\prime} \in N$, then $q$ is conjugate to $q^{\prime}$ along some geodesic.

Remark. There are some examples of case 2(b). Consider $S^{n-1} \times R$, $S^{n-1} \times S^{1}, \mathbf{R P}^{n-1} \times R$, and $\mathbf{R} \mathbf{P}^{n-1} \times S^{1}$ where the $R$ (resp. $S^{1}$ ) is the subset $N$. We will study this case further in the next section with $M$ is homogeneous.

Proof. By Lemma 1.1 only (1) and (2) can happen. Let us consider (1) first.

By Corollary 1.4 we see that $M$ is diffeomorphic to $\mathbf{R}^{n}$, homeomorphic to $\mathbf{R P}^{n}$, or is one of the spaces in Warner's theorem. We need only show that the cases (b), (c), and (d) of Warner's theorem cannot hold. By Lemma 1.1 and Proposition 1.5 there is a 2-dimensional subspace $S \subset T_{p} M$ such that $d \circ f_{A T C}(S)=3$. Thus by Lemma 1.1 for every 3-dimensional subspace $S^{\prime}$ such that $S \subset S^{\prime}$ we have $d \circ f_{A T C}\left(S^{\prime}\right) \leqslant d \circ f_{A T C}(S)+1=4$. Therefore by Proposition 1.6 for every $X \in T_{q}\left(N_{S}^{A T C}\right)$ there is a number $C_{X}$ such that $Y \in\left(T_{q}\left(N_{S}^{A T C}\right)\right)^{\perp} \Rightarrow R(Y, X) X=C_{X} Y$. If $\gamma$ is a geodesic from $p$ lying in $N_{S}^{A T C}$, let $\mathcal{G}$ be the space of Jacobi fields $J(t)$ along $\gamma$ such that $J(0)=0$ and

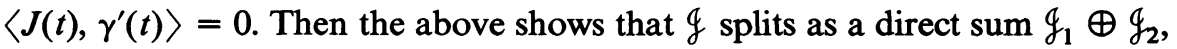
where $\mathscr{G}_{1}$ has dimension $n-3$ and consists of those Jacobi fields $J$ such that $J(t) \in\left(T_{\gamma(t)} N_{S}^{A T C}\right)^{\perp}$, and $\mathscr{q}_{2}$ has dimension 2 and consists of those Jacobi fields $J$ with $J(t) \in T_{\gamma(t)} N_{S}^{A T C}$. Further, as in the argument of Theorem 3.2, since $R\left(Y, \gamma^{\prime}(t)\right) \gamma^{\prime}(t)=c(t) Y$ for all $Y \in\left(T_{\gamma(t)} N_{S}^{A T C}\right)^{\perp}$, we know that if for some $t_{0}$ there is a $J_{0} \in \mathscr{g}_{1}$ such that $J_{0}\left(t_{0}\right)=0$ (and $J_{0} \neq 0$ ), then for all $J \in \mathscr{g}_{1}, J\left(t_{0}\right)=0$. Now if $J \in \mathcal{g}$ then $J=J_{1}+J_{2}$ where $J_{1} \in \mathcal{g}_{1}$ and $J_{2} \in$ $g_{2}$, and if $J\left(t_{0}\right)=0$ then $J_{1}\left(t_{0}\right)=J_{2}\left(t_{0}\right)=0$ since $J_{1}$ and $J_{2}$ are independent whenever they are not 0 . Thus we have shown that the first conjugate point along $\gamma$ has multiplicity $1,2, n-3, n-2$, or $n-1$. This eliminates cases (c) and (d) of Warner's theorem. Now we consider case (b), that is, we assume that the first conjugate locus consists entirely of points of multiplicity 1. For $n>4$ the above argument shows that for any geodesic $\gamma \subset N_{S}^{A T C}, \gamma(0)=p$, the Jacobi fields vanishing at the first conjugate locus come from $q_{2}$. Thus the first conjugate point in $M$ along $\gamma$ is also the first conjugate point in $N_{S}^{A T C}$ along $\gamma$, and therefore $N_{S}^{A T C}$ is a compact 3-dimensional riemannian manifold such that the first conjugate locus to $p$ is spherical and consists of conjugate points of order 1. Further, since the cut locus is equal to the first conjugate locus, we know that $N_{S}^{A T C}$ is simply connected. But Warner's theorem says 
this cannot happen. Thus we can assume $n=4$. By the above argument we know that there is some geodesic $\gamma \subset N_{S}^{A T C}, \gamma(0)=p$, such that the Jacobi fields $J$ which vanish at the first conjugate point are precisely those $J$ 's in $g_{1}$. By continuity this must be true along every such $\gamma$. Now by Lemma 1.1 and Proposition 5.5 of [1], $\left\{S \subset T_{p} M \mid \operatorname{dim} S=2\right.$ and $\left.d \circ f_{A T C}(S)=3\right\}$ is open. Let $X, Y$ be an orthogonal basis for $S$. Choose $\tilde{S}$ in the above set so that $X, \tilde{Y}$ is an orthonormal basis for $\tilde{S}$ when $\tilde{Y}$ is chosen close to $Y$ but $\tilde{Y} \notin T_{p} N_{S}^{A T C}$. Let $\gamma$ be the geodesic determined by $X$. Then $\gamma \subset N_{S}^{A T C}$ and $\gamma \subset N_{S}^{A T C}$. Let $\tilde{g}_{1} \oplus \tilde{g}_{2}=\mathcal{G}$ be the decomposition of $\mathcal{g}$ with respect to $N_{S}^{A T C}$. By the above argument if $J \in \tilde{g}_{1}$, then $J$ vanishes at the first conjugate point along $\gamma$. Thus the space of Jacobi fields vanishing at the first conjugate point along $\gamma$ contains both $\mathscr{g}_{1}$ and $\tilde{g}_{1}$. But these are distinct 1-dimensional subspaces of $\mathscr{g}$ contradicting the assumption that the conjugate point had multiplicity 1 . Thus (1) is completed.

Now we consider (2). Let $\mathbb{Q}=\left\{S \subset T_{p} M \mid \operatorname{dim} S=1\right.$ and $d \circ f_{A T C}(S)=$ 2\}. We know by Lemma 1.1 and Proposition 5.5 of [1] that $\mathcal{Q}$ is open in $G_{1}(M)$. Let $\tilde{S}=\cap_{s \in \mathbb{Q}} f_{A T C}(S)$. For dimension reasons $\tilde{S}$ can be seen as a finite intersection $f_{A T C}\left(S_{1}\right) \cap f_{A T C}\left(S_{2}\right) \cap f_{A T C}\left(S_{3}\right)$. The subspace $\tilde{S}$ is precisely the subspace $K$ defined in Theorem 2.8 , although Theorem 2.8 does not apply when $n=4$. We will use this in the next section.

We claim that $\tilde{S}$ is 1-dimensional, and if $S_{1}, S_{2} \in \mathbb{Q}$ such that $S_{2} \not \subset f_{A T C} S_{1}$ then $\tilde{S}=f_{A T C}\left(S_{1}\right) \cap f_{A T C}\left(S_{\tilde{S}}\right)$.

To prove the claim let $\tilde{\tilde{S}}=f_{A T C}\left(S_{1}\right) \cap f_{A T C}\left(S_{2}\right)$ for $S_{\tilde{s}}, S_{2} \in \mathbb{Q}$ and $S_{2} \not$ $f_{A T C} S_{1}$. We will show that $\tilde{\tilde{S}}$ is 1-dimensional, and $\tilde{\tilde{S}} \subset f_{A T C}(S)$ for any $S \in \mathcal{Q}$. By Lemma $1.1, d \circ f_{A T C}\left(S_{1}+S_{2}\right) \leqslant 3$. Further, since $d \circ f_{A T C} S_{1}=2$, $S_{2} \not \subset f_{A T C} S_{1}$, and $S_{2}, f_{A T C} S_{1}$ are contained in $f_{A T C}\left(S_{1}+S_{2}\right)$, we see that $d \circ f_{A T C}\left(S_{1}+S_{2}\right)=3$. Now $f_{A T C}\left(S_{1}\right) \subset f_{A T C}\left(S_{1}+S_{2}\right)$ and $f_{A T C}\left(S_{2}\right) \subset$ $f_{A T C}\left(S_{1}+S_{2}\right)$ and they are 2-dimensional. Thus $f_{A T C}\left(S_{1}\right) \cap f_{A T C}\left(S_{2}\right)$ is 1-dimensional $\left(f_{A T C}\left(S_{1}\right) \neq f_{A T C}\left(S_{2}\right)\right.$ since $S_{2} \subset f_{A T C}\left(S_{2}\right)$ and $\left.S_{2} \not \subset f_{A T C}\left(S_{1}\right)\right)$. The above argument shows that for arbitrary $S, S^{\prime} \in \mathbb{Q}$ the dimension of $f_{A T C}(S)$ $\cap f_{A T C}\left(S^{\prime}\right)$ is either 1 or 2 . Now choose $S_{3} \in \mathbb{Q}$ such that $S_{3} \not \subset f_{A T C}\left(S_{1}+\right.$ $\left.S_{2}\right)$. This can be done since $Q$ is open and the dimension of $f_{A T C}\left(S_{1}+S_{2}\right)=$ 3 , while the dimension of the manifold is $\geqslant 4$. Now the argument above shows that $f_{A T C}\left(S_{3}\right) \cap f_{A T C}\left(S_{1}\right)$ is 1-dimensional, and $f_{A T C}\left(S_{3}\right) \cap f_{A T C}\left(S_{2}\right)$ is 1-dimensional. On the other hand, $f_{A T C}\left(S_{3}\right) \cap f_{A T C}\left(S_{1}+S_{2}\right)$ is at most 1-dimensional. Since $f_{A T C}\left(S_{1}\right) \subset f_{A T C}\left(S_{1}+S_{2}\right)$ and $f_{A T C}\left(S_{2}\right) \subset f_{A T C}\left(S_{1}+S_{2}\right)$, we see that $f_{A T C}\left(S_{3}\right) \cap f_{A T C}\left(S_{1}\right)=f_{A T C}\left(S_{3}\right) \cap f_{A T C}\left(S_{1}\right)=f_{A T C}\left(S_{1}\right) \cap f_{A T C}\left(S_{2}\right)=$ $\tilde{\tilde{S}}$. Thus we have shown that for $S \in \mathbb{Q}, S \not \subset f_{A T C}\left(S_{1}+S_{2}\right) \Rightarrow \stackrel{\approx}{S} \subset f_{A T C}(S)$. What if $S \in \mathbb{Q}$ and $S \subset f_{A T C}\left(S_{1}+S_{2}\right)$ ? In this case $f_{A T C}(S) \subset$ $f_{A T C}\left(S_{1}+S_{2}\right)$. Thus $f_{A T C}\left(S_{3}\right) \cap f_{A T C}(S) \subset f_{A T C}\left(S_{3}\right) \cap f_{A T C}\left(S_{1}+S_{2}\right)=\tilde{S}$. 
Since $f_{A T C}\left(S_{3}\right) \cap f_{A T C}(S)$ is 1-dimensional, $f_{A T C}\left(S_{3}\right) \cap f_{A T C}(S)=\tilde{\tilde{S}}$. Thus $\tilde{\tilde{S}}$ $\subset f_{A T C}(S)$, and the claim follows.

Now let $N=N_{S}^{A T C}$. We know that the dimension of $N \geqslant 1$, and that on the other hand $N \subset N_{S_{1}}^{A T C} \cap N_{S_{2}}^{A T C}$ which is one-dimensional. Thus $N$ is 1-dimensional, and $N$ is the component of $N_{S_{1}}^{A T C} \cap N_{S_{2}}^{A T C}$ containing $p$. Now case (b) holds if for every $q, q^{\prime} \in N, q$ is conjugate to $q^{\prime}$ along some geodesic. So assume that there exists $q, q^{\prime} \in N$ such that $q$ is not conjugate to $q^{\prime}$ along any geodesic. Now $N$ is a one-dimensional connected totally geodesic submanifold. Thus $N$ is a geodesic $\gamma$ through $p$. Since $N$ is almost totally convex and $q$ is not conjugate to $q^{\prime}$ along any geodesic, $\gamma$ is the only geodesic from $q$ to $q^{\prime}$. (By this we mean for example that if $\gamma$ is a closed geodesic then any geodesic from $q$ to $q^{\prime}$ is just some turns around $\gamma$.) To complete the theorem we need only show that the path space $\Omega_{q, q^{\prime}}$ has the homotopy type of a C.W. complex with no cells in dimensions 1 through $n-2$. To do this, by the Morse theory [5], it is sufficient to show that any point of $\gamma$ conjugate to $q$ along $\gamma$ has multiplicity $n-1$. By a previous argument it is sufficient to show $R\left(Y, \gamma^{\prime}(t)\right) \gamma^{\prime}(t)=c(t) Y$ for all $Y \in\left(\gamma^{\prime}(t)\right)^{\perp} \subset T_{\gamma(t)} M$.

We claim that for all $Y \in\left(\gamma^{\prime}(t)\right)^{\perp} \subset T_{\gamma(t)} M$ we have $R\left(Y, \gamma^{\prime}(t)\right) \gamma^{\prime}(t)=$ $c(t) Y$.

To prove the claim we recall that $\gamma=N_{S_{1}}^{A T C} \cap N_{S_{2}}^{A T C}$ (actually the connected component). Since $S_{1}, S_{2} \in \mathbb{Q}$ by Proposition 1.6 we have $R\left(Y, \gamma^{\prime}(t)\right) \gamma^{\prime}(t)=c^{i}(t) Y$ for all $Y \in\left(T_{\gamma(t)} N_{S_{i}}^{A T C}\right)^{\perp}, i=1$, 2. Since $N_{S_{1}+S_{2}}^{A T C}$ has dimension 3 and the dimension of the manifold is $>4$, we see that there is a $Y$ in $\left(T_{\gamma(t)} N_{S_{1}}^{A T C}\right)^{\perp} \cap\left(T_{\gamma(t)} N_{S_{2}}^{A T C}\right)^{\perp} \supset\left(T_{\gamma(t)} N_{S_{1}+S_{2}}^{A T C}\right)^{\perp}$. Thus $c^{1}(t)=c^{2}(t)$. Let $c(t)=c^{1}(t)=c^{2}(t)$. By the linearity of $R\left(-, \gamma^{\prime}(t)\right) \gamma^{\prime}(t)$ we see that $R\left(Y, \gamma^{\prime}(t)\right) \gamma^{\prime}(t)=c(t) Y$ for all $Y$ in $\left(T_{\gamma(t)} N_{S_{1}}^{A T C}\right)^{\perp}+\left(T_{\gamma(t)} N_{S_{2}}^{A T C}\right)^{\perp}=$ $\left(T_{\gamma(t)} N_{S^{1}}^{A T C} \cap T_{\gamma(t)} N_{S^{1}}^{A T C}\right)^{\perp}=\left(\gamma^{\prime}(t)\right)^{\perp}$. Hence the claim follows, and the proof of the theorem is complete.

\section{Homogeneous spaces}

In this section we consider riemannian manifolds $M^{n}$ whose isometry groups $I(M)$ are transitive. In particular we are concerned with the cases $A T C_{p}(M)=n-1$ and $A T C_{p}(M) \geqslant \frac{1}{2}(n+3)$. We begin by extending Theorem 2.8 .

4.1. Theorem. Let $M$ be a homogeneous riemannian manifold with (transitive) group of isometries $G$. If $A T C_{p}(M)>\frac{1}{2}(n+3)$ for some $p \in M$ (and hence for all $p \in M)$, then there are $a G$-invariant distribution $D$ and an $\varepsilon>0$ 
such that for all $p \in M$ we have

(i) $D(p) \subset K(p)$ where $K(p)$ is the subspace of Theorem 2.8;

(ii) $D(p)$ is completely $\varepsilon$-geodesic;

(iii) if $q \in M$ such that $d(p, q)<\varepsilon$ and $\gamma$ is the unique minimal geodesic from $q$ to $p$, then $D(q) \subset \gamma^{\prime}(q)+\gamma_{q}^{p} D(p)$, where $\gamma_{q}^{p}$ is the parallel translation from $p$ to $q$.

Proof. By Theorem 2.8 the distribution $K(p)$ is $G$-invariant and completely $i(M)$-geodesic where $i(M)$ is the injectivity radius.

Let $\varepsilon_{1}=\frac{1}{2} i(M)$, and fix $p \in M$. For each $q \in B_{\varepsilon_{1}}(p)$ let $K_{q}^{p}=\gamma_{p}^{q}(K(q))+$ $\gamma^{\prime}(0)$, where $\gamma$ is the unique minimal geodesic from $p$ to $q$.

We claim that each $K_{q}^{p}$ is completely $\varepsilon_{1}$-geodesic. Since $K(q)$ is completely $2 \varepsilon_{1}$-geodesic, so is $K(q)+\gamma^{\prime}(q)$. Let $K_{q}^{p} \subset Q$ and $Q^{\prime}=\gamma_{q}^{p} Q$. Since $K(q)+$ $\gamma^{\prime}(q) \subset Q^{\prime}, \operatorname{Exp}_{q}\left(Q^{\prime} \cap B_{2 \varepsilon_{1}}(0)\right) \equiv N_{2 \varepsilon_{1}}\left(Q^{\prime}\right)$ is totally geodesic. Since $\gamma \subset$ $N_{2 \varepsilon_{1}} Q^{\prime}$, we have $p \in N_{2 \varepsilon_{1}}\left(Q^{\prime}\right)$. Since $N_{2 \varepsilon_{1}} Q^{\prime}$ is totally geodesic, $T_{p}\left(N_{2 \varepsilon_{1}}\left(Q^{\prime}\right)\right)=$ $Q$. Now $\operatorname{Exp}_{p}\left(Q \cap B_{\varepsilon_{1}}(0)\right) \subset N_{2 \varepsilon_{1}} Q^{\prime}$ and since they have the same dimension, $\operatorname{Exp}_{p}\left(Q \cap B_{\varepsilon_{1}}(0)\right)$ is totally geodesic. Hence the claim follows.

Now define

$$
D_{1}(p)=\left[\bigcap_{q \in B_{e_{1}}(p)} K_{q}^{p}\right] \cap K(p) .
$$

For dimension reasons the intersection can be taken to be finite, i.e.,

$$
D_{1}(p)=K(p) \cap K_{q_{1}}^{p} \cap K_{q_{2}}^{p} \cap \cdots \cap K_{q_{r}}^{p} .
$$

We also claim that $D_{1}(p)$ is completely $\varepsilon_{1}$-geodesic. Clearly $K(p)$ is completely $\varepsilon_{1}$-geodesic. The preceding claim gives $K_{q_{i}}^{p}$ is completely $\varepsilon_{1}$-geodesic. Now $\operatorname{dim}(K(p)) \leqslant n-A T C$ and $\operatorname{dim}\left(K_{q_{i}}^{p}\right) \leqslant n-A T C+1$, thus $\operatorname{dim}(K(p)$ $\left.+K_{q_{i}}^{p}\right) \leqslant 2 n-2 A T C+1<n-2$. Therefore by Proposition 2.4 the intersection is completely $\varepsilon_{1}$-geodesic.

We can define $D_{1}(q)$ for all $q \in M$ in the same way. Thus we get a $G$-invariant distribution $D_{1}$ such that $D_{1}(p) \subset K(p)$, and $D_{1}(p)$ is completely $\varepsilon_{1}$-geodesic for all $p \in M$.

Now let $\varepsilon_{i}=\frac{1}{2} \varepsilon_{i-1}$, and let $D_{i}$ be constructed from $D_{i-1}$ in the same way that $D_{1}$ was constructed from $K$. For dimension reasons there is some $j>0$ such that $D_{j}(p)=D_{j-1}(p)$. Let $D=D_{j}$ and $\varepsilon=\varepsilon_{j}$. Properties (i) and (ii) are immediate. Let $p \in M$ and $q \in B_{\varepsilon}(p)$. We have $D(q)=D_{j}(q)=$ $\left[\cap_{q^{\prime} \in B_{e j}(q)} D_{j-1}\left(q^{\prime}\right)_{q^{\prime}}^{q}\right] \cap D_{j-1}(q)$.

But $D(q)=D_{j}(q)=D_{j-1}(q)$, so

$$
D(q)=\left[\bigcap_{q^{\prime} \in B_{g}(q)} D\left(q^{\prime}\right)_{q^{\prime}}^{q}\right] \cap D(q) .
$$


Thus $D(q) \subset \bigcap_{q^{\prime} \in B_{\varepsilon^{\prime}(q)}} D\left(q^{\prime}\right)_{q^{\prime}}^{q}$. In particular $D(q) \subset D(p)_{p}^{q}=\gamma_{q}^{p} D(p)+$ $\gamma^{\prime}(q)$. Hence the theorem follows.

Remark. The author strongly suspects that $D(p)=K(p)$, i.e., $K(p)$ satisfies (iii) for $\varepsilon=i(M)$. We will eventually see that for simply connected normal homogeneous spaces this is, in fact, true.

4.2. Corollary. If $\operatorname{ATC}_{p}(M)=\frac{1}{2}(n+3)$, then either such a distribution $D$ exists or

$$
\begin{aligned}
& M \stackrel{\text { diffeo }}{\approx} \mathbf{R}^{n}, \\
& M \stackrel{\text { homeo }}{\approx} S^{n}, \\
& M \stackrel{\text { homeo }}{\approx} \mathbf{R P}^{n} .
\end{aligned}
$$

Proof. This follows from Corollary 2.9 and the proof of Theorem 4.1.

In the following theorem we will assume that $M$ is a normal homogeneous space. The property of normal homogeneous spaces, which we will use, is that: Every geodesic in $M$ is an orbit in $M$ of some one-parameter group of isometries.

4.3. Theorem. Let $M^{n}$ be a connected normal homogeneous space. If for some $p \in M$ (hence for all $p \in M) A T C_{p}(M)>\frac{1}{2}(n+3)$, then $\tilde{M} \stackrel{\text { iso }}{\simeq} M^{1} \times$ $M^{2}$, where $M^{1}$ is a simply connected manifold of constant curvature. Further $\operatorname{ATC}_{\tilde{p}}(\tilde{M}) \geqslant \operatorname{dim} M^{1} \geqslant \operatorname{ATC}_{p}(M)>\frac{1}{2}(n+3)$.

Proof. Consider the invariant distribution $D$ of Theorem 4.1. We claim that $D$ is invariant under holonomy.

In order to show $D$ is invariant under holonomy it is sufficient to show that the form $d$ defining $D$ is parallel. By the invariance (under isometries) of $d$ it is sufficient to show $\nabla_{X} d=0$ for all $X \in T_{p} M$ (some fixed $p$ ). To show this we need only show it on a basis $X_{1}, \cdots, X_{n}$ of $T_{p} M$. Choose an orthonormal basis $X_{1}, \cdots, X_{r}, X_{r+1}, \cdots, X_{n}$ such that $X_{1}, \cdots, X_{r} \in D(p)^{\perp}$ and $X_{r+1}, \cdots, X_{n} \in D(p)$.

Case 1. Let $r+1 \leqslant i \leqslant n$, and let $\gamma$ be the geodesic determined by $X_{i}$. To show $\nabla_{X_{i}} d=0$, it is sufficient to show that for $t<\varepsilon$ ( $\varepsilon$ of Theorem 4.1) $\gamma_{t}^{0} D(p)=D(\gamma(t))$. By (ii) of Theorem 4.1, $D(p)$ is completely $\varepsilon$-geodesic. Thus $N_{\varepsilon}(D(p)) \equiv \operatorname{Exp}_{p}\left(D(p) \cap B_{\varepsilon}(0)\right)$ is totally geodesic, and so for $t<\varepsilon$, $\gamma(t) \in N_{e}(D(p))$ and $\gamma_{t}^{0} D(p)=T_{\gamma(t)} N_{e}(D(p))$. By property (iii), $D(\gamma(t)) \subset$ $\gamma_{t}^{0} D(p)+\gamma^{\prime}(t)=T_{\gamma(t)}\left(N_{e} D(p)\right)=\gamma_{t}^{0} D(p)$. For dimension reasons $D(\gamma(t))=$ $\gamma_{t}^{0} D(p)$. Hence Case 1 is done.

Case 2. $1 \leqslant i \leqslant r$. Let $\gamma$ be the geodesic determined by $X_{i}$. Let $g_{t}$ be a one-parameter group of isometries such that $g_{t}(p)=\gamma(t)$. In particular for fixed $t<\varepsilon$ we have $g_{t *} \gamma^{\prime}(0)=\gamma^{\prime}(t)$. Again we will show that for $t<$ $\varepsilon, \gamma_{t}^{0}(D(p))=D(\gamma(t))$. Now since $g_{t *}: T_{p} M \rightarrow T_{\gamma(t)} M$ is an isometry, and 
$g_{t *}(D(p))=D(\gamma(t))$, and $g_{t *} \gamma^{\prime}(0)=\gamma^{\prime}(t)$, and $\gamma^{\prime}(0)=X_{i} \in(D(p))^{\perp}$, we see that $\gamma^{\prime}(t) \in D(\gamma(t))^{\perp}$. On the other hand, $\gamma^{\prime}(t) \in\left(\gamma_{t}^{0}(D(p))\right)^{\perp}$. Now by property (iii) of Theorem 4.1, $D(\gamma(t)) \subset \gamma_{t}^{0}(D(p))+\gamma^{\prime}(t)$. Thus $D(\gamma(t))=$ $\gamma_{t}^{0} D(p)$, and the claim follows.

Now let $\tilde{D}$ be the induced distribution on $\tilde{M}$. Since $\tilde{D}$ must also be invariant under holonomy, the de Rham splitting theorem tells us that $\tilde{M} \approx M^{1} \times M^{2}$, where $M^{2}$ is a totally geodesic submanifold through $p$ with $T_{p} M^{2}=D(p)$, while $M^{1}$ is the totally geodesic submanifold through $p$ with $T_{p} M^{1}=D(p)^{\perp}$. Since $\tilde{M}$ is simply connected, $M^{1}$ must be simply connected. Now, since $D(p)$ is completely $\varepsilon$-geodesic, Proposition 2.3 tells us that $M^{1}$ is a space of constant curvature. Now by (i) of Theorem 4.1, $D(p) \subset K(p)$. Thus by Theorem $2.8, \operatorname{dim} D(p) \leqslant n-A T C_{p}(M)$, and therefore $\operatorname{dim}\left(M^{1}\right) \geqslant$ $A T C_{p}(M)>\frac{1}{2}(n+3)$. On the other hand Proposition 3.4 of [1] gives $A T C_{\tilde{p}}(\tilde{M}) \geqslant \max \left\{A T C_{p_{1}}\left(M^{1}\right), A T C_{p_{2}}\left(M^{2}\right)\right\} \geqslant \operatorname{dim} M^{1}$. Hence the theorem follows.

4.4. Corollary. Let $M^{n}$ be a connected normal homogeneous space. If for some $p \in M$ we have $A T C_{p}(M)=\frac{1}{2}(n+3)$, then the same conclusions follow or else

$$
\begin{aligned}
& M \stackrel{\text { diffeo }}{\approx} \mathbf{R}^{n}, \\
& M \stackrel{\text { homeo }}{\approx} S^{n}, \\
& M \stackrel{\text { homeo }}{\approx} \mathbf{R P}^{n} .
\end{aligned}
$$

Proof. Immediate from the proof of the theorem and previous corollaries.

4.5. Corollary. Let $G$ be a Lie group which admits a bi-invariant metric, and $H$ a compact subgroup of $G$ such that $G$ effectively on $M^{n}=G / H$. If $\operatorname{dim} H>1 / 8(n+3)(3 n-5)$, then $\tilde{M} \approx M^{1} \times M^{2}$ where $M^{1}$ is diffeomorphic to $\mathbf{R}^{q}$ or $S^{q}$ and $q>\frac{1}{2}(n+3)$.

Proof. Put a bi-invariant metric on $G$. This metric will induce an invariant metric on $M$ such that the projection $G \rightarrow M$ is a riemannian submersion. With this metric, $M$ is a normal homogeneous space. $H$ acts on $M$ as a group of isometries which leave the point $e H$ fixed. The dimension assumption on $H$ along with Theorem 1.6 of [1] gives $A T C_{p}(M)>\frac{1}{2}(n+3)$. The result now follows from Theorem 4.3.

Remark. The result should hold with a slightly less restrictive condition on the dimension of $H$. This is true since the inequality of Theorem 1.6 of [1] can be improved in many cases.

4.6. Corollary. Assume that in Theorem 4.3 we also had $f_{A T C}(D(p))=$ $D(p)$. Then the deck transformations of the universal covering $\tilde{M} \stackrel{\pi}{\rightarrow} M$ are of the form $\left(f_{1}, f_{2}\right)$, where $\tilde{M}=M^{1} \times M^{2}$ as in the theorem, and $f_{i}$ is an isometry of $M^{i}$. 
If $M^{1}$ has constant nonpositive curvature, then $f_{1}$ is always the identity, and $M$ is isometric to $M^{1} \times M^{3}$ where $M^{2} \rightarrow M^{3}$ is the universal cover.

If $M^{1}$ has constant positive curvature, then $f_{1}$ is always either the identity or the antipodal map.

Remark. The assumption $f_{A T C}(D(p))=D(p)$ holds if $K(p)=D(p)$. The author suspects that this is always true.

Proof. Since the distribution $T_{p} M^{2}$ is the lift of the distribution $D(p)$, it must be preserved by the deck transformations. Thus if $f$ is a deck transformation, its differential at a point $p$ must look like the differential of an isometry $\left(f_{1}, f_{2}\right)$. Now since an isometry is determined by its differential at a point, $f=\left(f_{1}, f_{2}\right)$.

Let $(x, y) \in M^{1} \times M^{2}$ and $\left(x_{1}, y_{1}\right)=f(x, y)$. The proof will be complete if we show that $x$ is conjugate to $x_{1}$ along every geodesic in $M^{1}$ from $x$ to $x_{1}$.

Let $\gamma$ be a geodesic in $M^{1}$ from $x$ to $x_{1}$. Let $\tau(t)=\left(\gamma(t), y_{1}\right)$ be the corresponding geodesic in $\tilde{M}$ from $\left(x, y_{1}\right)$ to $\left(x_{1}, y_{1}\right)$. Let $\sigma(t)=\pi \circ \tau(t)$ be the corresponding geodesic in $M$ from $\pi\left(x, y_{1}\right)$ to $\pi\left(x_{1}, y_{1}\right)=\pi(x, y)$. Since $A T C_{p}(D(p))=D(p)$ for all $p$, we see that $N_{D\left(\pi\left(x, y_{1}\right)\right)}^{A T C}=\operatorname{Exp}_{\pi\left(x, y_{1}\right)} D\left(\pi\left(x, y_{1}\right)\right)$ $=\pi \circ \operatorname{Exp}_{\left(x, y_{1}\right)} T_{\left(x, y_{1}\right)} M^{2}=\pi\left(M^{2}\right)$ where $M^{2}$ is the copy of $M^{2}$ going through $\left(x, y_{1}\right)$. In particular both $\pi(x, y)$ and $\pi\left(x, y_{1}\right)$ lie in this $A T C$ submanifold while $\sigma(t)$ does not. Thus $\sigma(0)$ is conjugate to $\sigma(1)$ along $\sigma, \tau(0)$ is conjugate to $\tau(1)$ along $\tau$, and $\gamma(0)$ is conjugate to $\gamma(1)$ along $\gamma$. Hence the corollary follows.

Now consider homogeneous spaces $M$ which are not necessarily normal. If $A T C_{p}(M)>\frac{1}{2}(n+3)$, then the proof of Case 1 of Theorem 4.3 along with the fact that $D(p)$ is $\varepsilon$-geodesic shows that $D$ is an involutive distribution and that the leaves of the induced foliation are totally geodesic submanifolds (not necesarily embedded). Also, since $D$ is $I(M)$ invariant, the leaves are $I(M)$ invariant.

Now consider the distribution $A(p)=f_{A T C} D(p)$ (as before this is likely to be $D(p))$. Clearly $A(p) \subset K(p)$, so the dimension of $A(p)$ is less than or equal to $n-A T C_{p}$. It is not hard to see that $A(p)$ is an $I(M)$-invariant involutive distribution, and that the leaves of the induced foliation are the embedded totally geodesic $A T C$ submanifolds $N_{A(p)}^{A T C}$. Fix $p \in M$, let $H \subset I(M)$ be the subgroup leaving $p$ fixed, and let $\tilde{H} \subset I(M)$ be the (closed) subgroup which takes $p$ to some point on the leaf $N_{A(p)}^{A T C}$ through $p$. We thus see that $A(p)$ induces a fibration:

$$
\begin{aligned}
& M=\frac{I(M)}{H} \leftarrow \frac{\tilde{H}}{H} \\
& \frac{\stackrel{\downarrow}{I(M)}}{\tilde{H}}
\end{aligned}
$$


Further if the fibration is trivial, then $A(p)=\{0\}$, and therefore $D(p)=$ $\{0\}$. Hence $M$ is a space of constant curvature, and we have shown

4.7. Proposition. Let $M$ be a connected riemannian homogeneous space such that $A T C_{p}(M)>\frac{1}{2}(n+3)$ at some point. Then $M$ is the total space of a fibration whose fibres are totally geodesic embedded ATC submanifolds. Further if the fibration is trivial, then $M$ is a space of constant curvature.

We now wish to consider the case $A_{T C}(M)=n-1$ in the homogeneous setting. The previous results tell us a lot about this case. However, some more can be said in the non-normal case.

4.8. Proposition. Let $M^{n}$ be a connected riemannian homogeneous space with $n \geqslant 5$. If $A T C_{p}(M)=n-1$ for $p \in M$, then the universal covering space $\tilde{M}$ is $(n-2)$-connected.

Proof. Consider Theorem 3.6. The above proposition holds in Cases 1 and 2(a). Thus we need only consider Case 2(b). As mentioned in the proof of Theorem 3.6 the subpsace $\tilde{S}_{q}$ is precisely the subspace $K(q)$. Thus the distribution $K$ is one-dimensional. So the distribution $D$ defined in Theorem 4.1 is either equal to $K$ or 0 -dimensional. If it is 0 -dimensional, then $M$ is a space of constant curvature, so the result holds. Thus we may assume $D(p)=K(p)=\tilde{S}_{p}$.

Let $T \subset T_{p} M$ be a two-dimensional subspace. By Proposition 2.3 there are constants $C^{\prime \prime}$ and $C^{\perp}$ such that $k(T)=C^{\prime \prime}$ if $\tilde{S} \subset T$, and $k(T)=C^{\perp}$ if $T$ is perpendicular to $\tilde{S}$, where $k$ is the sectional curvature. Thus for general $T$ we have $k(T)=C_{1}^{2} C^{\prime \prime}+C_{2}^{2} C^{\perp}$ where $C_{1}^{2}+C_{2}^{2}=1$. ( $T$ has as orthonormal basis $Z_{1}, Z_{2}$ where $Z_{1} \in \tilde{S}^{\perp}$ and $Z_{2}=C_{1} Y_{1}+C_{2} Y_{2}$ with $Y_{1} \in \tilde{S}^{\perp}, Y_{2} \in \tilde{S}$. Now apply Proposition 2.3 to the cross terms.)

We claim that if $T$ is a two-dimensional subspace of $T_{p} M$ such that $\tilde{S} \subset T$, then $\operatorname{Exp}_{p}(T)=N_{T}^{A T C}$ which is a two-dimensional space of constant curvature $C^{\prime \prime}$.

Let $Z \in T$ with $Z$ perpendicular to $\tilde{S}$. Since $A T C_{p}(M)=n-1$, $d \circ f_{A T C}(T) \leqslant 3$. Assume $d \circ f_{A T C}(M)=3$. In case $(2 \mathrm{~b})$ of Theorem 3.6 we know that the set of 1-dimensional spaces $S$ with $d \circ f_{A T C}(S)=2$ is open. Choose such an $S$ such that $f_{A T C}(T) \cap S=\{0\}$. Let $T^{\prime}=S+Z$. Then $d \circ f_{A T C}\left(T^{\prime}\right) \leqslant 3$. Now by the definition of $\tilde{S}$ we have $\tilde{S} \subset f_{A T C}(S) \subset$ $f_{A T C}\left(T^{\prime}\right)$, which implies that $\tilde{S} \subset f_{A T C}\left(T^{\prime}\right)$ and $Z \in f_{A T C}\left(T^{\prime}\right)$, so that $T \subset$ $f_{A T C}\left(T^{\prime}\right)$. Thus $f_{A T C}(T) \subset f_{A T C}\left(T^{\prime}\right)$. For dimension reasons $f_{A T C}(T)=$ $f_{A T C}\left(T^{\prime}\right)$ but $S \not f_{A T C}(T)$ and $S \subset f_{A T C}\left(T^{\prime}\right)$, a contradiction. Thus $d \circ f_{A T C}(T)=2, T=f_{A T C}(T)$, and $\operatorname{Exp}_{p} T=N_{T}^{A T C}$.

To show $N_{T}^{A T C}$ has constant curvature $C^{\prime \prime}$ we need only show that $\tilde{S} \subset$ $T_{q} N_{T}^{A T C}$ for all $q \in N_{T}^{A T C}$, but this follows from Theorem 4.1(iii) since $\tilde{S}_{q}^{q}=D(q)$. 
We also claim that in the case we are considering (i.e., Case (2b) of Theorem 3.6), $C^{\prime \prime} \leqslant 0$. Assume $C^{\prime \prime}>0$, fix $p \in M$, and let $N=\operatorname{Exp}_{p} \tilde{S}$. In our case we know there is a $q \in N, q$ close to $p$ such that $q$ is conjugate to $p$ along some geodesic $\gamma$ not lying in $N$. Let $T=\gamma^{\prime}(0)+\tilde{S}$. Then $\gamma$ is a geodesic lying not in $N$ but in $N_{T}^{A T C}$, a 2-dimensional space of constant positive curvature $C$. But $\gamma$ is a geodesic from $p$ to $q$ where $q$ is close to $p$ and $\gamma \not \subset N$. This cannot happen in a 2-dimensional space of constant positive curvature.

The theorem will follow from the Morse theory [6] when we show:

We claim that all conjugate points have multiplicity greater than or equal to $n-2$.

Let $p \in M$, and $\gamma$ be any geodesic from $p$. Let $T=\gamma^{\prime}(0)+\tilde{S}$. Then since $\operatorname{Exp}_{p} T$ is totally geodesic, the space $g$ of Jacobi fields $J$ along $\gamma$ such that $J(0)=0,\left\langle J(t), \gamma^{\prime}(t)\right\rangle=0$, splits as a product $\mathscr{g}_{1} \oplus \mathscr{g}_{2}$, where $J \in \mathscr{g}_{1}$ satisfies $J(t) \in T_{\gamma(t)} N_{T}^{A T C}$, and $J \in \mathscr{g}_{2}$ satisfies $J(t) \in\left(T_{\gamma(t)} N_{T}^{A T C}\right)^{\perp}$. Now since $C^{\prime \prime} \leqslant$ 0 , no Jacobi field in $g_{1}$ ever vanishes. We need only show that if $J(t)=0$ for some $J \in g_{2}$, then $J(t)=0$ for all $J \in g_{2}$. To show this, we need only show that for each $t$ there is a $C(t)$ such that $K(s)=C(t)$ for all sections $s$ with $\gamma^{\prime}(t) \in s$ and $Y \in s$ for some $Y \in\left(T_{\gamma(t)} N_{T}^{A T C}\right)^{\perp}$. Now fix $t$. Then $\gamma^{\prime}(t)$ can be written as $C_{1} Z+C_{2} V$ where $C_{1}^{2}+C_{2}^{2}=1,\|Z\|=1,\|V\|=1, V \in \tilde{S}_{\gamma(i)} \subset$ $T_{\gamma(t)} N_{T}^{A T C}$, and $Z \in \tilde{S}_{\gamma(t)}^{\perp} \subset T_{\gamma(t)} N_{T}^{A T C}$. Thus for any such $s$, using Proposition 2.3, we have $K(s)=\left\langle R\left(\gamma^{\prime}(t), Y\right) Y, \gamma^{\prime}(t)\right\rangle=C_{1}^{2} C^{\perp}+C_{2}^{2} C^{\prime \prime}$. Hence the result follows. q.e.d.

If $M$ is a homogeneous riemannian manifold such that $A T C_{p}(M)=n-1$ and $n \geqslant 5$, then either $K(p)=D(p)$ (both are one-dimensional) or $M$ is a space of constant curvature. Thus if $M$ is a normal homogeneous space of nonconstant curvature both Theorem 4.3 and Corollary 4.6 apply. In this case it is not hard to see that the group of isometries of $M$ has dimension $\frac{1}{2} n(n-1)+1$. The riemannian manifolds $M$, along with the isometry groups $I(M)$ such that the dimension of $I(M)$ is $\frac{1}{2} n(n-1)+1$, have been classified (see [3]). One example, $S^{n-1} \times{ }_{K} S^{1}$ where $K=\{i d, f\}$ with $f=$ (antipodal, antipodal) was mistakenly left off this list. This example shows up in consequence of Corollary 4.6.

One also sees in [3] that manifolds $M$ with $\operatorname{dim} I(M)=\frac{1}{2} n(n-1)+1$ are precisely those homogeneous riemannian manifolds such that $\operatorname{dim} I_{p}=$ $\frac{1}{2}(n-1)(n-2)$. One can show, with an argument similar to Proposition 1.5 and some Lie theory that if $\operatorname{dim} I_{p}=\frac{1}{2}(n-1)(n-2)$ and $n \geqslant 5$, then $A T C_{p}(M)=n-1$.

The interest in studying the case $\operatorname{dim} I_{p}=\frac{1}{2}(n-1)(n-2)$ comes from the fact that for $n \neq 4$ the largest proper subgroup of $S O(n)$ has dimension 
$\frac{1}{2}(n-1)(n-2),[3]$. As mentioned before the homogeneous case has essentially been done, while Theorem 3.6 gives us some geometric information in the nonhomogeneous case.

\section{References}

[1] C. Croke, Some new Riemannian invariants, J. Differential Geometry 15 (1980), 443-466.

[2] J. Cheeger \& D. G. Ebin, Comparison theorems in Riemannian geometry, North Holland, Amsterdam, 1975.

[3] S. Kobayashi, Transformation groups in differential geometry, Springer, Berlin, 1972.

[4] L. N. Mann, Highly symmetric homogeneous spaces, Canad. J. Math. 26 (1974) 291-293.

[5] J. Milnor, Morse theory, Annals of Math. Studies of. 51, Princeton University Press, Princeton, 1963.

[6] F. W. Warner, The conjugate locus of a Riemannian manifold, Amer. J. Math. 87 (1965) 575-604.

[7] Conjugate loci of constant order, Ann. of Math. 86 (1967) 192-212. 
